\title{
Abundances in planetary nebulae: NGC 1535, NGC 6629, He2-108, and Tc1 ^
}

\author{
S. R. Pottasch ${ }^{1}$, R. Surendiranath ${ }^{2}$, and J. Bernard-Salas ${ }^{3,4}$ \\ ${ }^{1}$ Kapteyn Astronomical Institute, PO Box 800, 9700 AV Groningen, The Netherlands \\ e-mail: pottasch@astro.rug.nl \\ 2 T-1, 10/5, 2nd Cross, 29th Main, BTM I Stage, Bangalore-560068, India ${ }^{\star \star}$ \\ 3 Institut d'Astrophysique Spatiale, Paris-Sud 11, 91405 Orsay, France \\ ${ }^{4}$ Center for Radiophysics and Space Research, Cornell University, Ithaca, NY 14853, USA
}

Received 7 February 2011 / Accepted 14 April 2011

\section{ABSTRACT}

\begin{abstract}
Context. Models have been made of stars of a given mass that produce planetary nebulae that usually begin on the AGB (although they may begin earlier) and run to the white dwarf stage. While these models cover the so-called dredge-up phases when nuclear reactions occur and the newly formed products are brought to the surface, it is important to compare the abundances predicted by the models with the abundances actually observed in PNe.

Aims. The aim of the paper is to determine the abundances in a group of PNe with uniform morphological and kinematic properties. The PNe we discuss are circular with rather low-temperature central stars and are rather far from the galactic plane. We discuss the effect these abundances have on determining the evolution of the central stars of these PNe.

Methods. The mid-infrared spectra of the planetary nebulae NGC 1535, NGC 6629, He2-108, and Tc1 (IC 1266) taken with the Spitzer Space Telescope are presented. These spectra were combined with the ultraviolet IUE spectra and with the spectra in the visual wavelength region to obtain complete, extinction-corrected spectra. The chemical composition of these nebulae is then found by directly calculating and adding individual ion abundances. For two of these PNe, we attempted to reproduce the observed spectrum by making a model nebula. This proved impossible for one of the nebulae and the reason for this is discussed. The resulting abundances are more accurate than earlier studies for several reasons, the most important is that inclusion of the far infrared spectra increases the number of observed ions and makes it possible to include the nebular temperature gradient in the abundance calculations.

Results. The abundances of the above four PNe have been determined and compared to the abundances found in five other PNe with similar properties studied earlier. These abundances are further compared with values predicted by the models of Karakas (2003). From this comparison we conclude that the central stars of these PNe originally had a low mass, probably between $1 M_{\odot}$ and $2.5 M_{\odot}$. A further comparison is made with the stellar evolution models on the HR diagram, from which we conclude that the core mass of these PNe is between $0.56 M_{\odot}$ and $0.63 M_{\odot}$.

Conclusions. A consistent picture of the evolution of this group of $\mathrm{PNe}$ is found that agrees with the predictions of the models concerning the present nebular abundances, the individual masses, and luminosities of these PNe. The distance of these PNe can be determined as well.
\end{abstract}

Key words. ISM: abundances - infrared: ISM

\section{Introduction}

The evolution of planetary nebulae $(\mathrm{PNe})$ has been studied for about four decades with the result that a general picture of the evolution is understood, but the details are still being debated. This is both because many of the parameters involved are poorly known and because the atmosphere of the central star is difficult to describe, including the effective temperature, radius, and mass loss rate. The (in many cases) uncertain distance enhances these difficulties. Furthermore, the interaction of the central star with the nebula is often unclear. This manifests itself in a difficulty explaining the ionization structure in some PNe.

The abundances of some elements change in the course of evolution of the central star. We discuss here primarily the elements helium, nitrogen, carbon, and oxygen, although neon, argon, sulfur, chlorine, and sometimes other elements are

\footnotetext{
* Based on observations with the Spitzer Space Telescope, which is operated by the Jet Propulsion Laboratory, California Institute of Technology.

$\star \star$ Formerly with the Indian Institute of Astrophysics, Bangalore.
}

determined as well. These elements are ejected by the star and are measured in the nebula. These abundances provide complementary information which aid in understanding the evolution and which can eventually confirm or limit the results of models of the evolution.

We are concerned here with the abundances found in $\mathrm{PNe}$ whose central stars are rather bright and whose morphology is usually described as round or elliptical. We present new abundances for four such nebulae: NGC 1535, NGC 6629, Tc1 (IC 1266), and He2-108. The abundances in these nebulae will be combined with the results found earlier in five other PNe, which are also excited by bright stars and which have similar (round or elliptical) morphology. These PNe have similar spatial properties as well: they are at rather high galactic latitudes. We can therefore speak of a class of nebulae. This class has been extensively studied earlier but from a different point of view. The central stars of these PNe are among the brightest known in the visible. It is therefore possible to observe these spectra with very high resolution. The profiles of hydrogen and helium lines can then be analyzed with the initial goal of obtaining the effective 
temperature $\left(T_{\text {eff }}\right)$ and gravity of these stars. The final goal is to use these quantities to study the mass and luminosity (thus the evolution) of these stars, but additional information is required to do this.

One of the early extensive studies of the line profiles is that of Mendez et al. (1988, 1992). These authors take high resolution optical spectra of $24 \mathrm{PNe}$ and interpret these spectra using NLTE plane-parallel static model atmospheres which contain only hydrogen and helium. The values of $T_{\text {eff }}$ and the stellar helium abundance are determined by fitting the observed HeI and HeII line profiles while the gravity $(\log g)$ is found by fitting the hydrogen line profiles (usually $\mathrm{H} \gamma$ but sometimes $\mathrm{H} \beta$ ). Then, making use of theoretical evolution diagrams which plot $T_{\text {eff }}$ against $\log g$ for different values of the stellar mass $M_{\mathrm{s}} / M_{\odot}$, the value of $M_{\mathrm{S}} / M_{\odot}$ is determined. Using this mass and the value of gravity, the angular radius of the exciting star is determined. Combining this value with the previously found temperature and the observed magnitude corrected for extinction, the distance to the nebulae is found. In the course of time this group has improved the model atmospheres used by first including the sphericity of the atmosphere (Kudritzki et al. 1997) and then by including other elements besides $\mathrm{H}$ and $\mathrm{He}$ in the atmosphere (Kudritzki et al. 2006). These improved models do not change the stellar masses or PNe distances substantially. Both the masses and the distances determined in this way, however, are suspect. Quoting Kudritzki et al. (2006) "the masses determined seem systematically larger than white dwarf masses and some of the objects ... have unrealistically high masses". Concerning the distances: for the 18 cases where the distance thus found may be compared with statistical distance (Stanghellini et al. 2008; and Cahn et al. 1992) they are always higher, in 11 cases more than $60 \%$ higher.

Recently Pauldrach et al. (2004) used a different approach. By including models losing mass they are able to use "observed" mass loss rates and terminal velocities to obtain the stellar masses and distances. These masses and distances are even higher than found by Kudritzki et al. (2006) and are shown to be implausibly high by Napiwotzki (2006). Two arguments are used. Because high mass central stars are probably descended from high mass progenitors they should have the properties of high mass stars. First they should belong to the disk population of the galaxy which is characterized by small scale heights perpendicular to the galactic disk. Secondly such high mass star should have produced and dredged up substantial amounts of both nitrogen and helium. Napiwotzki concludes that on both of these points the high masses found by Pauldrach et al. (2004) are unlikely.

This suggests the following step: the abundances of those elements produced by the various stars should be accurately determined. These abundances may then be used to determine the mass of the star in question by comparing the observed abundance with the abundances determined by nucleosynthesis model calculations made in the course of evolution of stars of different masses. For these models, the calculations made by Karakas (2003) are used. These masses can then be compared with those found by Kudritzki et al. (2006). Because the masses used in the calculations of Karakas are the initial stellar masses, and the masses given by Kudritzki et al. (2006) are the present (almost final) stellar masses, a ratio between the initial and final mass must be known. For this secondary information the values given by Weidemann (2000) will be used. Unfortunately the uncertainties present in each of these steps will accumulate so that definite conclusions are difficult to draw. It is however important that central star masses can be derived from accurate nebular abundances.
The main purpose of this paper is to obtain accurate abundances for four of the nebulae discussed by Mendez et al. (1992). The most important reason that this can be achieved is the inclusion of the mid-infrared spectrum taken with the IRS spectrograph of the Spitzer Space Telescope (Werner et al. 2004). The reasons for this have been discussed in earlier papers (e.g. see Pottasch \& Beintema 1999; Pottasch et al. 2000, 2001; Bernard Salas et al. 2001), and can be summarized as follows: 1) the intensity of the infrared lines is not very sensitive to the electron temperature nor to possible extinction effects; 2) use of the infrared line intensities enable a more accurate determination of the electron temperature for use with the visual and ultraviolet lines; 3 ) the number of observed ionization stages is doubled.

For two of the PNe a second method of determining the abundances is attempted using a nebular model. This has several advantages. First it provides a physical basis for the electron temperature determination. Secondly it permits abundance determination for elements which are observed in only one, or a limited number of ionic stages. This is true of $\mathrm{Mg}, \mathrm{Fe}, \mathrm{P}$ and $\mathrm{Cl}$, which would be less reliably determined without a model. A further advantage of modeling is that it provides information on the central star and other properties of the nebula.

A disadvantage of modeling is that there are more unknowns than observations and some assumptions must be made especially concerning the geometry and the form of the radiation field of the exciting star. The difficulties we encountered in determining a model for NGC 1535 are discussed below.

Abundance determination for these nebulae have been made earlier and will be compared with our values in the individual sections.

This paper is structured as follows. In Sects. 2-5 the observations of NGC 1535, NGC 6629, Tc1 and He2-108 will be discussed and the resultant abundances will be given and compared to earlier results. In Sect. 7, the models for NGC 1535 and Tc1 are presented and discussed. In Sect. 8 the evolutionary state of the nebulae is discussed. Finally, our conclusions are given in Sect. 9.

\section{NGC 1535}

NGC 1535 (PN G206.4-40.5) is a roughly circular nebula consisting of a bright inner ring of about $20^{\prime \prime} \times 17^{\prime \prime}$ surrounded by a much fainter outer shell of $48^{\prime \prime} \times 42^{\prime \prime}$ (Banerjee \& Anandaro 1991). Tylenda et al. (2003) list a size of $33^{\prime \prime} \times 32^{\prime \prime}$ down to the $10 \%$ level. The nebula is at a very high galactic latitude. The distance to the nebula is rather uncertain. Ciardullo et al. (1999) give a value of $2.3 \mathrm{kpc}$ on the basis of a possible association of a nearby star with the PN. Herald \& Bianchi (2004) use a value of $1.6 \mathrm{kpc}$ on the basis of nebular models. We shall use the latter value when necessary for two reasons. First we feel that the evidence for association of the star and nebula is rather weak. Secondly at the larger distance the dimensions of the nebula are rather large for such a bright nebula. We stress however that the distance is uncertain.

\subsection{The infrared spectrum}

Observations of NGC 1535 were made using the Infrared Spectrograph (IRS, Houck et al. 2004) on board the Spitzer Space Telescope with AOR keys of 4111616 (on target) and 4111872 (background). The reduction started from the droop images which are equivalent to the most commonly used Basic Calibrated Data $(b c d)$ images but lack stray-cross removal and 
Table 1. IRS spectra of the four nebulae.

\begin{tabular}{|c|c|c|c|c|c|c|c|c|c|}
\hline Identification & $\lambda(\mu \mathrm{m})$ & $\begin{array}{l}\text { NGC 1535 } \\
\text { Intensity }\end{array}$ & $\begin{array}{c}\text { NGC } 1535 \\
\mathrm{I} / \mathrm{H} \beta\end{array}$ & $\begin{array}{c}\text { Tc1 } \\
\text { Intensity }\end{array}$ & $\begin{array}{c}\mathrm{Tc} 1 \\
\mathrm{I} / \mathrm{H} \beta\end{array}$ & $\begin{array}{c}\mathrm{He} 2-108 \\
\text { Intensity }\end{array}$ & $\begin{array}{c}\mathrm{He} 2-108 \\
\mathrm{I} / \mathrm{H} \beta\end{array}$ & $\begin{array}{l}\text { NGC 6629 } \\
\text { Intensity } \\
\end{array}$ & $\begin{array}{c}\text { NGC } 6629 \\
\text { I/H } \beta\end{array}$ \\
\hline $\mathrm{F}$ & 6.48 & & & $198 \pm 27$ & 3.2 & & & & \\
\hline [Ni II] ? & 6.63 & & & $76.5 \pm 8$ & 1.24 & & & & \\
\hline [Ar II] & 7.026 & & & $2020 \pm 70$ & 32.8 & & & & \\
\hline $\mathrm{H}_{\mathrm{I}}(6-5)$ & 7.47 & $36.2 \pm 2.1$ & & $230 \pm 17$ & & $40.7 \pm 4.8$ & & $306 \pm 29$ & \\
\hline $\mathrm{F}$ & 7.757 & & & & & & & $37.2 \pm 13$ & 0.44 \\
\hline $\mathrm{F}$ & 8.497 & & & $384 \pm 10$ & & & & & \\
\hline [Ar III] & 8.99 & $76.5 \pm 4.3$ & 3.32 & $399 \pm 16$ & 6.50 & $299 \pm 13$ & 25 & $1080 \pm 45$ & \\
\hline [S IV $]$ & 10.512 & $915 \pm 35$ & 39.8 & $29.2 \pm 2.2$ & 0.47 & $41.0 \pm 2.4$ & 3.6 & $1220 \pm 46$ & 14.4 \\
\hline Н I $(9-7)$ & 11.305 & & & $20.6 \pm 3$ & & & & $32.4 \pm 2.3$ & \\
\hline [Cliv] & 11.763 & $10.5 \pm 1.9$ & 0.455 & & & & & & \\
\hline Н г $(7-6+11-8)$ & 12.375 & $22.4: \pm 2.1$ & & $67.9 \pm 3.6$ & & $11.7 \pm 1.2$ & & $95.8 \pm 4.3$ & \\
\hline$\left[\mathrm{Ne}_{\mathrm{II}}\right]$ & 12.81 & $9.55 \pm 1.9$ & 0.415 & $2301 \pm 200$ & 37.5 & $1175 \pm 130$ & 102 & $839 \pm 19$ & 102 \\
\hline [Ne III] & 15.555 & $1920 \pm 40$ & 83.5 & $89.5 \pm 5$ & 1.46 & $189 \pm 6$ & 16.5 & $7530 \pm$ & 89.1 \\
\hline $\mathrm{F}$ & 17.66 & & & & & $35 \pm 12$ & 3 & & \\
\hline$[\mathrm{P}$ III] & 17.88 & & & & & & & $51 \pm 2.3$ & 0.61 \\
\hline [S III] & 18.714 & $67.4 \pm 3.3$ & 2.93 & $863 \pm 80$ & 14.0 & $830 \pm 150$ & 72.2 & $1540 \pm 32$ & 18.2 \\
\hline [Cl IV] & 20.316 & $10.8 \pm 0.9$ & 0.47 & & & & & & \\
\hline [Ar III] & 21.820 & $7.44 \pm 0.73$ & 0.323 & $26.6 \pm 2$ & 0.432 & $19.4 \pm 2$ & 1.69 & $85.4 \pm 6.9$ & 1.01 \\
\hline$[\mathrm{Fe}$ III] & 22.92 & & & $18.1 \pm 1.6$ & 0.295 & $11.7 \pm 0.7$ & 1.02 & & \\
\hline [O IV $]$ & 25.889 & $2466 \pm 21$ & 107 & & & & & & \\
\hline [S III] & 33.48 & $63.2 \pm 1.9$ & 2.75 & $382 \pm 33$ & 6.21 & $402 \pm 18$ & 35.0 & $807 \pm 34$ & 6.3 \\
\hline$[\mathrm{Ne}$ III] & 36.013 & $203 \pm 2.2$ & 8.84 & & & & & $531 \pm 24$ & 6.3 \\
\hline
\end{tabular}

Notes. The measured line intensity is given in Cols. 3, 5, 7 and 9. Columns 4, 6, 8 and 10 give the ratio of the line intensity to $\mathrm{H} \beta(=100)$. (†) Intensities measured in units of $10^{-14} \mathrm{erg} \mathrm{cm}^{-2} \mathrm{~s}^{-1}$. The intensities SH measurements (below $19 \mu \mathrm{m}$ ) and the SL measurements have been increased by a factor to bring them all to the scale of the LH measurements. The factors used are given in text. Lines identified by "F" are related to the Fullerine molecule (2010).

flat-field. The data were processed using the s15.3 version of the pipeline and using a script version of Smart (Higdon et al. 2004). The tool irsclean was used to remove rogue pixels. The different cycles for a given module were combined to enhance the $\mathrm{S} / \mathrm{N}$. Then the resulting high-resolution modules (HR) were extracted using full aperture measurements, and the SL measurements setting a window column extraction. This same reduction has been used for all nebulae discussed in this paper.

The IRS high resolution spectra have a spectral resolution of about 600, which is a factor of between 2 and 5 less than the resolution of the ISO SWS spectra. The mid-infrared measurements are made with several different diaphragm sizes. Because the diaphragms are smaller than the size of the nebulae and are all of differing size, we first discuss how the different spectra are placed on a common scale.

Two of the three diaphragms used have high resolution: the short high module (SH) measures from $9.9 \mu \mathrm{m}$ to $19.6 \mu \mathrm{m}$ and the long high module (LH) from $18.7 \mu \mathrm{m}$ to $37.2 \mu \mathrm{m}$. The $\mathrm{SH}$ has a diaphragm size of $4.7^{\prime \prime} \times 11.3^{\prime \prime}$, while the LH is $11.1^{\prime \prime} \times$ $22.3^{\prime \prime}$. If the nebulae are uniformly illuminating then the ratio of the intensities would simply be the ratio of the areas measured by the two diaphragms. Since this is not so, we may use the ratio of the continuum intensity in the region of wavelength overlap at $19 \mu \mathrm{m}$. These continua are equal when the $\mathrm{SH}$ intensities are increased by a factor of 3.2. The third diaphragm is a long slit which is $4^{\prime \prime}$ wide and extends over the entire nebula. This SL module measures in low resolution and measures between $5.5 \mu \mathrm{m}$ and $14 \mu \mathrm{m}$. These spectra are normalized by making the lines in common between the SL and SH modules agree. Especially important is the agreement of the [S IV] line at $10.51 \mu \mathrm{m}$. A uniform scale is obtained by increasing the SH intensities by a factor of 3.2 with respect to the LH intensities. The SL intensities are increased by a factor of 1.23 .
The IRS measurement of NGC 1535 was centered at $\operatorname{RA}(2000) 04^{\mathrm{h}} 14^{\mathrm{m}} 15.9^{\mathrm{s}}$ and $\operatorname{Dec}(2000)-12^{\circ} 44^{\prime} 21^{\prime \prime}$. This is almost exactly the same as the value measured by Kerber et al. (2003) of $\operatorname{RA}(2000) \quad 04^{\mathrm{h}} 14^{\mathrm{m}} 15.78^{\mathrm{s}}$ and $\operatorname{Dec}(2000)$ $-12^{\circ} 44^{\prime} 21.7^{\prime \prime}$, which is presumably the coordinate of the central star. Thus the IRS measurement was well centered on the nebula. The fluxes were measured using the Gaussian line-fitting routine. The measured emission line intensities are given in Table 1, after correcting the $\mathrm{SH}$ measurements by the factor 3.2 and the SL measurements by a factor of 1.23 , in the column labeled "intensity". The $\mathrm{H} \beta$ flux found from the infrared hydrogen lines (especially the lines at $12.37 \mu \mathrm{m}$ ) using the theoretical ratios of Hummer \& Storey (1987), is $2.3 \times 10^{-11} \mathrm{erg} \mathrm{cm}^{-2} \mathrm{~s}^{-1}$, which is about $49 \%$ of the total $\mathrm{H} \beta$ intensity. This is reasonable since the well-centered LH diaphragm covers a large fraction of the nebula. Note that by scaling in this way the nebula is assumed homogeneous when it is larger than the different modules.

\subsection{The visual spectrum}

The visual spectrum has been measured by at least six authors. We list here the results from four of these. The line intensities listed have been corrected by each author for a value of extinction determined by them to obtain a theoretically correct Balmer decrement. The result are listed in Table 2, where the last column lists the average value which we have used. No attempt has been made to use a common extinction correction because then the Balmer decrement will be incorrect. The value of extinction $\mathrm{C}$ which the individual authors found is listed at the bottom of the table. None of the spectra measure the weaker lines very well. The errors may be judged by the agreement (or disagreement) of the various measures and appear to be within $20 \%$ for the stronger lines and worse for the weaker lines. 
Table 2. Visual spectrum of NGC 1535.

\begin{tabular}{|c|c|c|c|c|c|c|}
\hline \multirow{2}{*}{$\begin{array}{l}\lambda \\
(\AA)\end{array}$} & \multirow[t]{2}{*}{ Ion } & \multicolumn{4}{|c|}{ Intensities ${ }^{\dagger}$} & \multirow{2}{*}{$\begin{array}{l}\text { Average } \\
\text { Intens. }\end{array}$} \\
\hline & & (1) & (2) & (3) & (4) & \\
\hline $3727^{*}$ & {$\left[\mathrm{O}_{\mathrm{II}}\right]$} & 4.1 & 7.1 & 8.41 & 9.37 & 8.41 \\
\hline 3869 & [Ne III] & 90 & 95.8 & 97 & 116 & 101 \\
\hline 4267 & $\mathrm{C}_{\mathrm{II}}$ & 0.4 : & 0.34 & 1.03 & 0.36 & 0.34 \\
\hline 4363 & [O III] & 13.3 & 12.5 & 11 & 12.6 & 12.6 \\
\hline 4686 & $\mathrm{He}_{\mathrm{II}}$ & 27 & 14.2 & 18.3 & 17 & 17.5 \\
\hline 4711 & [Ar IV] & 4.5 & 4.2 & 5.4 & 4.5 & 4.5 \\
\hline 4740 & [Ar IV] & 3.8 & 3.2 & 3.8 & 3.36 & 3.4 \\
\hline 4861 & $\mathrm{H} \beta$ & 100 & 100 & 100 & 100 & 100 \\
\hline 5007 & [O III] & 1220 & 1210 & 1180 & 1190 & 1200 \\
\hline 5517 & {$\left[\mathrm{Cl}_{\mathrm{III}}\right]$} & & 0.242 & 0.264 & & 0.25 \\
\hline 5538 & {$\left[\mathrm{Cl}_{\text {III }}\right]$} & & 0.177 & 0.175 & & 0.175 \\
\hline 5755 & {$\left[\mathrm{~N}_{\mathrm{II}}\right]$} & & $0.1:$ & 0.62 & & $0.1:$ \\
\hline 5876 & $\mathrm{He}_{\mathrm{I}}$ & 10.2 & 12.6 & 11.8 & 12.6 & 12.2 \\
\hline 6312 & [S III] & & 0.303 & & 0.28 & 0.30 \\
\hline 6584 & {$\left[\mathrm{~N}_{\text {III }}\right]$} & 1.8 & 1.62 & $0.25:$ & 0.85 & 1.6: \\
\hline 6717 & {$[\mathrm{~S}$ II $]$} & & 0.0627 & & & 0.0627 \\
\hline 6731 & {$\left[\mathrm{~S}_{\text {II }}\right]$} & & 0.114 & & & 0.114 \\
\hline 7135 & [Ar III] & 6.0 & 6.42 & 5.3 & & 6.3 \\
\hline 7263 & [Ar IV] & & 0.217 & & & 0.217 \\
\hline 8045 & [Cl IV $]$ & & 0.512 & 0.53 & & 0.52 \\
\hline 9532 & {$\left[\mathrm{~S}_{\mathrm{III}}\right]$} & & 6.75 & & & 6.75 \\
\hline $\mathrm{C}(\mathrm{H} \beta)$ & & 0.2 & 0.07 & 0.11 & 0.01 & \\
\hline
\end{tabular}

Notes. ${ }^{(\dagger)}$ References: (1) Barker (1989); (2) Milingo et al. (2010); (3) Aller \& Czyzak (1979); (4) Krabbe \& Copetti (2006). (:) Indicates uncertain values. $\left.{ }^{*}\right)$ This is a blend of $\lambda 3726$ and $\lambda 3729$ lines. Only Aller \& Czyzak are able to resolve this doublet: $3726=5.4,3729=3.01{ }^{(C)}$ is the extinction used by the author.

\subsection{The ultraviolet spectrum}

Quite a large number of IUE spectra of this nebula have been taken. If only those taken with the large diaphragm are counted there are six shortwavelength spectra and five longwavelength spectra with the central star included. These have been made with low resolution. Also two shortwavelength spectra (SWP 10821 and 13495) and one longwavelength spectrum (LWR02165) were taken with high resolution. They also included the central star. Two spectra are also available which included only the nebula (SWP15497 and LWR11975). These spectra are especially useful because longer exposures can be made without saturating the stronger lines. The $\mathrm{N}$ III line can only be seen on these spectra. The IUE diaphragm is an ellipse about $10^{\prime \prime} \times 21^{\prime \prime}$; the wavelength range is from $1150 \AA$ to about $3220 \AA$. The high resolution spectra have a resolution of $0.2 \AA$ while the low resolution is about $6 \AA$. There are also two spectra taken with the Hopkins Ultraviolet Telescope (HUT), one taken with the central star in the diaphragm, the other has only the nebula in the $9.4^{\prime \prime} \times 116^{\prime \prime}$ diaphragm. The HUT spectra have a resolution of about $3 \AA$ and they cover the wavelength range between $830 \AA$ and $1860 \AA$.

Because the nebula is in all cases larger than the diaphragm used and the various spectra are centered at different positions in the nebula, a total spectrum is obtained by normalizing the individual spectra to the strong He II line at $1640 \AA$. Thus as basis we use the high resolution IUE spectra SWP13495 and LWR02165; the other lines are found by using the measured ratio to the intensity of the $1640 \AA$ line in the individual spectra. The values are given in the third column of Table 3 . The measured values are then corrected for the diaphragm size and the extinction using the total $\mathrm{H} \beta$ flux of $4.73 \times 10^{-11} \mathrm{erg} \mathrm{cm}^{-2} \mathrm{~s}^{-1}$ (see below), then
Table 3. UV spectrum of NGC 1535.

\begin{tabular}{|c|c|c|c|c|}
\hline \multirow{2}{*}{$\begin{array}{l}\lambda \\
(\AA)\end{array}$} & \multirow[t]{2}{*}{ Ion } & \multicolumn{3}{|c|}{ Intensities } \\
\hline & & 1 & 2 & $(\mathrm{I} / \mathrm{H} \beta)$ \\
\hline 977 & $\mathrm{C}_{\text {IIII }}$ & 9.6 & 8.4 & 18 \\
\hline 1175 & $\mathrm{C}_{\text {III] }}$ & 34 & 23.5 & 50 \\
\hline 1548 & $\mathrm{C}_{\text {IV }}$ & 30.8 & 19.3 & 40.7 \\
\hline 1550 & $\mathrm{C}_{\text {IV }}$ & 16.8 & 10.0 & 21.2 \\
\hline 1640 & Не II & 95 & 56.4 & 119 \\
\hline 1661 & O III] & 1.8 & 1.1 & 2.3 \\
\hline 1663 & $\mathrm{O}_{\text {III] }}$ & 6.9 & 4.1 & 8.7 \\
\hline 1678 & $?$ & 4.8 & 2.8 & 6.0 \\
\hline 1750 & $\mathrm{~N}_{\text {III] }}$ & 8.5 & 5.05 & 10.7 \\
\hline 1906 & $\mathrm{C}_{\text {III] }}$ & 85.2 & 50.9 & 108 \\
\hline 1909 & $\mathrm{C}_{\text {IIII] }}$ & 58.0 & 34.5 & 73 \\
\hline 2297 & $\mathrm{C}_{\text {IIII] }}$ & 16 & 9.5 & 20 \\
\hline 2734 & $\mathrm{He}_{\text {II }}$ & 3.5 & 1.9 & 4.0 \\
\hline 2837 & {$\left[\mathrm{Fe}_{\mathrm{IV}}\right] ?$} & 1.8 & 1.0 & 2.1 \\
\hline 3048 & O III & 7.0 & 3.6 & 7.2 \\
\hline 3134 & $\mathrm{O}_{\text {III }}$ & 28.0 & 14.2 & 30 \\
\hline 3204 & He II & 7.2 & 3.7 & 7.7 \\
\hline
\end{tabular}

Notes. ${ }^{(1)}$ Measured intensity from in units of $10^{-13} \mathrm{erg} \mathrm{cm}^{-2} \mathrm{~s}^{-1}$. (2) Intensity corrected for diaphragm size and extinction in units of $10^{-12} \mathrm{erg} \mathrm{cm}^{-2} \mathrm{~s}^{-1}$. $\mathrm{I} / \mathrm{H} \beta$ is normalized to $\mathrm{H} \beta=100$.

the intensity of the He II at $4686 \AA$ becomes $8.25 \times 10^{-12} \mathrm{erg} \mathrm{cm}^{-2}$ (from Table 2). The theoretical helium spectrum (Hummer \& Storey 1987) then gives the intensity of the $1640 \AA$ line to be $5.64 \times 10^{-11} \mathrm{erg} \mathrm{cm}^{-2}$. The other lines are then corrected for their extinction with respect to the $1640 \AA$ line using the values given by Fluks et al. (1994) and the value $C=0.09$ as given below. These values are listed in Col. 4 of Table 3. In Col. 5 of the table the ratio of the line to $\mathrm{H} \beta$ normalized to $\mathrm{H} \beta=100$ is given. The uncertainties in the intensities are estimated to be $20 \%$ for the stronger lines and $30 \%$ for the weaker lines.

\subsection{Extinction}

The two methods which can be used for obtaining the extinction are: (1) comparison of radio emission with $\mathrm{H} \beta$ flux, and (2) comparison of observed and theoretical Balmer decrement. The four values of the extinction correction $C(\mathrm{H} \beta)$ which are found in the literature are given in Table 2 , and are seen to have a rather large range. Let us discuss the radio emission and the $\mathrm{H} \beta$ flux.

\subsubsection{The $6 \mathrm{~cm}$ radio emission and the $\mathrm{H} \beta$ flux}

The $6 \mathrm{~cm}$ flux density has been measured by Griffith et al. (1994) as $168 \mathrm{mJy}$. This corresponds to an $\mathrm{H} \beta$ flux of $4.73 \times$ $10^{-11}$ erg $\mathrm{cm}^{-2} \mathrm{~s}^{-1}$ using the electron temperature and helium abundance given below. The observed $\mathrm{H} \beta$ flux of $3.8 \times$ $10^{-11} \mathrm{erg} \mathrm{cm}^{-2} \mathrm{~s}^{-1}$ (see Cahn et al. 1992). This leads to an extinction constant of $C(\mathrm{H} \beta)=0.094$ or $E_{B-V}=0.064$. This is quite similar to the values found from the Balmer decrement and listed in Table 2. This value, together with the extinction curve of Fluks et al. (1994). has been used in correcting the UV fluxes in Table 3.

\section{Chemical composition of NGC 1535}

The method of analysis is the same as used in the papers cited in the introduction. First the electron density and temperature as function of the ionization potential are determined. Then the 
Table 4. Observed electron density indicators in the nebulae.

\begin{tabular}{|c|c|c|c|c|c|c|c|c|c|c|}
\hline Ion & $\begin{array}{c}\text { Ioniz. } \\
\text { Pot.(eV) }\end{array}$ & $\begin{array}{l}\text { Lines } \\
\text { Used }\end{array}$ & $\begin{array}{l}\text { Obs. Ratio } \\
\text { NGC } 1535\end{array}$ & $\begin{array}{l}N_{\mathrm{e}}\left(\mathrm{cm}^{-3}\right) \\
\text { NGC } 1535\end{array}$ & $\begin{array}{c}\text { Obs. Ratio } \\
\text { Tc1 }\end{array}$ & $\begin{array}{c}N_{\mathrm{e}}\left(\mathrm{cm}^{-3}\right) \\
\mathrm{Tc} 1\end{array}$ & $\begin{array}{c}\text { Obs. Ratio } \\
\text { He2-108 }\end{array}$ & $\begin{array}{c}N_{\mathrm{e}}\left(\mathrm{cm}^{-3}\right) \\
\mathrm{He} 2-108\end{array}$ & $\begin{array}{l}\text { Obs. Ratio } \\
\text { NGC } 6629\end{array}$ & $\begin{array}{l}N_{\mathrm{e}}\left(\mathrm{cm}^{-3}\right) \\
\text { NGC } 6629\end{array}$ \\
\hline$[\mathrm{S}$ II $]$ & 10.4 & $6731 / 6716$ & 1.8: & 2500: & 1.59 & 2900: & & & 0.74 & 1600 \\
\hline [O II] & 13.6 & $3726 / 3729$ & 1.8: & 3000: & 1.51 & 1900: & & & 1.63 & 2400 \\
\hline [S III] & 23.3 & $33.5 / 18.7$ & 0.94 & 700 & 0.444 & 3200 & 0.485 & 2200 & 0.525 & 2200 \\
\hline [Cl III] & 23.8 & $5538 / 5518$ & 0.70: & 600: & 1.06 & 3000: & & & $0.9:$ & 1400: \\
\hline [C III] & 24.4 & $1906 / 1909$ & 1.48 & 900 & 1.24 & 4500 & & & & \\
\hline [Ar IV] & 40.7 & $4740 / 4711$ & 0.76: & 1000: & & & & & & \\
\hline
\end{tabular}

Notes. (:) Indicates uncertain values.

Table 5. Observed electron temperature indicators in the nebulae.

\begin{tabular}{|c|c|c|c|c|c|c|c|c|c|c|}
\hline Ion & $\begin{array}{c}\text { Ioniz. } \\
\text { Pot.(eV) }\end{array}$ & $\begin{array}{l}\text { Lines } \\
\text { Used }\end{array}$ & $\begin{array}{l}\text { Obs. Ratio } \\
\text { NGC } 1535\end{array}$ & $\begin{array}{c}T_{\mathrm{e}}(\mathrm{K}) \\
\text { NGC } 1535\end{array}$ & $\begin{array}{c}\text { Obs. Ratio } \\
\text { Tc1 }\end{array}$ & $\begin{array}{l}T_{\mathrm{e}}(\mathrm{K}) \\
\mathrm{Tc} 1\end{array}$ & $\begin{array}{c}\text { Obs. Ratio } \\
\text { He2-108 }\end{array}$ & $\begin{array}{c}T_{\mathrm{e}}(\mathrm{K}) \\
\mathrm{He} 2-108\end{array}$ & $\begin{array}{l}\text { Obs. Ratio } \\
\text { NGC } 6629\end{array}$ & $\begin{array}{c}T_{\mathrm{e}}(\mathrm{K}) \\
\mathrm{NGC} 6629\end{array}$ \\
\hline$\left[\mathrm{N}_{\mathrm{II}}\right]$ & 14.5 & $5577 / 6584$ & & & 0.0114 & 9300 & & & & \\
\hline [S III] & 23.3 & $6312 / 18.7$ & 0.102 & 13000 & 0.0337 & 9100 & & & 0.029: & 8700: \\
\hline [Ar III] & 27.6 & $7135 / 8.99$ & 1.9 & 14000 & 0.888 & 9000 & 0.60 & 8000 & 1.0 & 8500 \\
\hline [Ar III] & 27.6 & $7135 / 5192$ & & & 190 & 9000 & & & & \\
\hline [O III] & 35.1 & $4363 / 5007$ & 0.0105 & 11800 & 0.00446 & 9000 & 0.0055 & 9500 & 0.0041 & 8700 \\
\hline [O III] & 35.1 & $1663 / 5007$ & 0.0092 & 10700 & & & & & & \\
\hline [Ne III] & 41.0 & $3868 / 15.5$ & 1.21 & 12200 & & & 0.285 & 8200 & 0.42 & 8900 \\
\hline
\end{tabular}

ionic abundances are determined, using density and temperature appropriate for the ion under consideration, together with Eq. (1). Then the element abundances are found for those elements in which a sufficient number of ion abundances have been derived.

\subsection{Electron density}

The ions used to determine $N_{\mathrm{e}}$ are listed in the first column of Table 4. The ionization potential required to reach that ionization stage, and the wavelengths of the lines used, are given in Cols. 2 and 3 of the table. Note that the wavelength units are $\AA$ when 4 ciphers are given and microns when 3 ciphers are shown. The observed ratio of the lines is given in the fourth column; the corresponding $N_{\mathrm{e}}$ is given in the fifth column. The temperature used is discussed in the following section, but is unimportant since these line ratios are essentially determined by the density.

The electron density appears to be about $1000 \mathrm{~cm}^{-3}$ although the two ions with the lowest ionization potential give a somewhat higher value. These values are less well determined because the ratios are poorly measured. The density is probably not uniform as indicated by the structures seen in the central area of the nebula, which may contribute to this difference. A density of $1000 \mathrm{~cm}^{-3}$ is used in the abundance determination in Table 6 , but none of the abundances listed in the table is sensitive to the density in the range shown in the table.

\subsection{Electron temperature}

A number of ions have lines originating from energy levels far enough apart that their ratio is sensitive to the electron temperature. These are listed in Table 5, which is arranged similarly to the previous table. While there is a slight scatter in these values there is no clear indication of a temperature gradient as function of the ionization potential as has been seen in some other nebulae. An electron temperature of $12000 \mathrm{~K}$ will be used with an uncertainty of less than $1000 \mathrm{~K}$.

\subsection{Ionic and element abundances}

The ionic abundances have been determined using the following equation:

$\frac{N_{\text {ion }}}{N_{\mathrm{p}}}=\frac{I_{\text {ion }}}{I_{\mathrm{H}_{\beta}}} N_{\mathrm{e}} \frac{\lambda_{\mathrm{ul}}}{\lambda_{\mathrm{H}_{\beta}}} \frac{\alpha_{\mathrm{H}_{\beta}}}{A_{\mathrm{ul}}}\left(\frac{N_{\mathrm{u}}}{N_{\mathrm{ion}}}\right)^{-1}$

where $I_{\text {ion }} / I_{\mathrm{H}_{\beta}}$ is the measured intensity of the ionic line compared to $\mathrm{H} \beta, N_{\mathrm{p}}$ is the density of ionized hydrogen, $\lambda_{\mathrm{ul}}$ is the wavelength of this line, $\lambda_{\mathrm{H}_{\beta}}$ is the wavelength of $\mathrm{H} \beta, \alpha_{\mathrm{H}_{\beta}}$ is the effective recombination coefficient for $\mathrm{H} \beta, A_{\mathrm{ul}}$ is the Einstein spontaneous transition rate for the line, and $N_{\mathrm{u}} / N_{\text {ion }}$ is the ratio of the population of the level from which the line originates to the total population of the ion. This ratio has been determined usually using a five level atom. Sometimes a two level atom was sufficient.

The results are given in Table 6, where the first column lists the ion concerned, and the second column the line used for the abundance determination. The third column gives the intensity of the line used relative to $\mathrm{H} \beta=100$. The fourth column shows the ionic abundances, and the fifth column gives the ionization correction factor (ICF). This has been determined empirically, usually by looking at the ionization potential of the missing ion. Notice that the ICF is unity for all elements except for Ar, S and $\mathrm{Cl}$ where it is close to unity. The helium abundance has been derived with the help of the theoretical work of Benjamin et al. (1999) and Porter et al. (2005).

\subsection{Comparison with other determinations}

In Table 7 the present abundances are compared to earlier determinations. The agreement is usually within a factor of 2, except for Chlorine which is difficult to measure.

\section{Tc1 (IC 1266)}

Tc1 (PN G345.2-08.8, also known as IC 1266, SaSt 2-16 and IRAS 17418-4604) is morphologically quite similar to 
Table 6. Ionic concentrations and chemical abundances in NGC 1535.

\begin{tabular}{lccccc}
\hline \hline Ion & $\lambda$ & Int./H $\beta$ & $N_{\text {ion }} / N_{\mathrm{p}}$ & ICF & $N_{\text {el. }} / N_{\mathrm{p}}$ \\
\hline $\mathrm{He}^{+}$ & 5875 & 12.2 & 0.076 & & \\
$\mathrm{He}^{++}$ & 4686 & 17.5 & 0.015 & 1 & 0.091 \\
$\mathrm{C}^{++}$ & 1909 & 181 & $1.23(-4)$ & & \\
$\mathrm{C}^{+3}$ & 1548 & 61.9 & $3.54(-5)$ & 1 & $1.6(-4)$ \\
$N^{+}$ & 6584 & 1.6 & $2.0(-7)$ & & \\
$N^{++}$ & 1750 & 10.7 & $3.2(-5)$ & 1 & $3.2(-5)$ \\
$\mathrm{O}^{+}$ & 3727 & 8.4 & $1.38(-6)$ & & \\
$\mathrm{O}^{++}$ & 5007 & 1200 & $2.4(-4)$ & & \\
$\mathrm{O}^{+3}$ & 25,9 & 107 & $2.03(-5)$ & 1 & $2.7(-4)$ \\
$\mathrm{Ne}^{+}$ & 12.8 & 0.42 & $5.2(-7)$ & & \\
$\mathrm{Ne}^{++}$ & 15.5 & 83.5 & $4.70(-5)$ & & \\
$\mathrm{Ne}^{++}$ & 3869 & 101 & $4.95(-5)$ & 1.1 & $5.4(-5)$ \\
$\mathrm{S}^{+}$ & 6731 & 0.114 & $3.7(-9)$ & & \\
$\mathrm{S}^{++}$ & 18.7 & 2.93 & $2.29(-7)$ & & \\
$\mathrm{S}^{+3}$ & 10.5 & 39.8 & $8.9(-7)$ & 1.2 & $1.3(-6)$ \\
$\mathrm{Ar}^{++}$ & 8.99 & 3.32 & $2.83(-7)$ & & \\
$\mathrm{Ar}^{++}$ & 7135 & 6.3 & $3.57(-7)$ & & \\
$\mathrm{Ar}^{+3}$ & 4740 & 3.4 & $5.75(-7)$ & 1.2 & $1.1(-6)$ \\
$\mathrm{Cl}^{++}$ & 5538 & 0.175 & $1.5(-8)$ & & \\
$\mathrm{Cl}^{+3}$ & 11.8 & 0.455 & $2.24(-8)$ & & \\
$\mathrm{Cl}^{+3}$ & 8045 & 0.52 & $3.36(-8)$ & 1.2 & $6.0(-8)$ \\
\hline
\end{tabular}

Notes. Wavelength in Angstrom for all values of $\lambda$ above 1000, otherwise in $\mu \mathrm{m}$. Intensities given with respect to $\mathrm{H} \beta=100$. (:) Indicates uncertain values.

Table 7. Comparison of abundances in NGC 1535.

\begin{tabular}{lcccccc}
\hline \hline Ele. & pres & bark & milin & AC & TPP & KC \\
\hline $\mathrm{He}$ & 0.091 & 0.097 & 0.0 .96 & 0.094 & 0.091 & 0.105 \\
$\mathrm{C}(-4)$ & 1.6 & 0.8 & 1.91 & 3.65 & & \\
$\mathrm{~N}(-5)$ & 3.2 & 4.3 & 2.09 & & & 1.3 \\
$\mathrm{O}(-4)$ & 2.7 & 3.3 & 2.98 & 4.05 & 3.8 & 2.6 \\
$\mathrm{~S}(-6)$ & 1.3 & & & & & \\
$\mathrm{Ar}(-6)$ & 1.1 & 1.2 & 0.937 & 1.93 & & \\
$\mathrm{Ne}(-5)$ & 5.3 & 7.1 & 6.37 & 8.3 & 9.5 & 7.3 \\
$\mathrm{Cl}(-7)$ & 0.60 & & 0.184 & 1.26 & & \\
\hline
\end{tabular}

References. bark: Barker (1989); milin: Milingo et al. (2010); AC: Aller \& Czyzak (1979); TPP: Torres-Peimbert \& Peimbert (1977); KC: Krabbe \& Copetti (2006).

NGC 1535. It is roughly circular and has a size at the $10 \%$ level of $12.9^{\prime \prime} \times 12.2^{\prime \prime}($ Tylenda et al. 2003). A somewhat smaller diameter $\left(9.6^{\prime \prime}\right)$ is given by Acker et al. (1992). The size is small enough so that most of the radiation can be measured in the IUE diaphragm. The nebula is surrounded by a faint halo which is also circular and has a diameter of about $53^{\prime \prime}$.

The $6 \mathrm{~cm}$ continuum radio flux density has been measured by Griffith et al. (1994) as 140 mJy. Milne \& Aller (1982) have measured the $2 \mathrm{~cm}$ radio flux density as $130 \mathrm{mJy}$, which corresponds to a value of $147 \mathrm{mJy}$ at $6 \mathrm{~cm}$. We use an average value of $145 \mathrm{mJy}$ at $6 \mathrm{~cm}$, which corresponds to an $\mathrm{H} \beta$ flux of $5.1 \times 10^{-11} \mathrm{erg} \mathrm{cm}^{-2} \mathrm{~s}^{-1}$. The measured $\mathrm{H} \beta$ flux is $2.18 \times 10^{-11} \mathrm{erg} \mathrm{cm}^{-2} \mathrm{~s}^{-1}$ (see Acker et al. 1992) which leads to an extinction coefficient $C=0.36$.

\subsection{Infrared spectrum}

The IRS measurement of Tc 1 was centered at RA(2000) $17^{\mathrm{h}} 45^{\mathrm{m}} 35.3^{\mathrm{s}}$ and $\operatorname{Dec}(2000)-46^{\circ} 05^{\prime} 23.3^{\prime \prime}$. This is almost exactly the same as the value measured by Kerber et al. (2003) of $\mathrm{RA}(2000) 17^{\mathrm{h}} 45^{\mathrm{m}} 35.3^{\mathrm{s}}$ and $\operatorname{Dec}(2000)-46^{\circ} 05^{\prime} 23.8^{\prime \prime}$, which is presumably the coordinate of the central star. Thus the IRS measurement was well centered on the nebula. The measured
Table 8. Visual spectrum of Tc 1.

\begin{tabular}{|c|c|c|c|c|}
\hline \multirow{2}{*}{$\begin{array}{l}\lambda \\
(\AA)\end{array}$} & \multirow[t]{2}{*}{ Ion } & \multicolumn{2}{|c|}{ Intensities $^{\dagger}$} & \multirow{2}{*}{$\begin{array}{c}\text { Average } \\
\text { Intens. }\end{array}$} \\
\hline & & (1) & (2) & \\
\hline 3726 & [O II] & 130 & & 130 \\
\hline 3729 & [O II] & 86 & & 86 \\
\hline 4340 & $\mathrm{H} \gamma$ & & 44.1 & 44.1 \\
\hline 4363 & [O III] & 0.555 & 0.45 & 0.55 \\
\hline 4471 & $\mathrm{He}_{\mathrm{I}}$ & & 1.1 & 1.1 \\
\hline 4861 & $\mathrm{H} \beta$ & 100 & 100 & 100 \\
\hline 5007 & [O III] & 124 & & 124 \\
\hline 5192 & [Ar III] & 0.0314 & & 0.314 \\
\hline 5517 & {$\left[\mathrm{Cl}_{\text {IIII }}\right.$} & 0.285 & & 0.285 \\
\hline 5538 & [Cl III] & 0.303 & & 0.303 \\
\hline 5755 & {$\left[\mathrm{~N}_{\mathrm{II}}\right]$} & 1.09 & & 1.09 \\
\hline 5876 & $\mathrm{He}_{\mathrm{I}}$ & & 9.01 & 9.01 \\
\hline 6312 & [S III] & 0.471 & & 0.471 \\
\hline 6584 & {$\left[\mathrm{~N}_{\mathrm{II}}\right]$} & 95.4 & 95.1 & 95.4 \\
\hline 6717 & [S II] & 2.20 & & 2.20 \\
\hline 6731 & {$[\mathrm{~S}$ II $]$} & 3.50 & & 3.50 \\
\hline 7135 & [Ar III] & 5.75 & 5.29 & 5.65 \\
\hline 9069 & [S III] & 13.0 & & 13.0 \\
\hline $\mathrm{C}(\mathrm{H} \beta)$ & & 0.33 & 0.40 & \\
\hline
\end{tabular}

Notes. ${ }^{(\dagger)}$ References; (1) Williams et al. (2008); (2) Kingsburgh et al. (1994). (:) Indicates uncertain values. ${ }^{(C)}$ Is the extinction used by the author.

emission line intensities are given in Table 1, after correcting the SH measurements by the factor 2.02 and the SL measurements by a factor of 2.35 , in the column labeled "intensity". The $\mathrm{H} \beta$ flux found from the infrared hydrogen lines (especially the lines at $12.37 \mu \mathrm{m}$ ) using the theoretical ratios of Hummer \& Storey (1987), is $6.15 \times 10^{-11} \mathrm{erg} \mathrm{cm}^{-2} \mathrm{~s}^{-1}$, which is about $20 \%$ higher than the total $\mathrm{H} \beta$ intensity. This indicates that the LH diaphragm measured the entire nebula. The measurement of a higher flux in the infrared is within the uncertainties of the various measurements. Two of the features in the table have been identified as belonging to the fullerene molecules (Cami 2010).

\subsection{Visual spectrum}

There are only a few visual spectra of Tc 1 . The best is the very good spectrum reported by Williams et al. (2008). These authors measured the nebula at two positions at either side of the central star, but carefully avoiding the star. They used a rectangular slit $2^{\prime \prime} \times 4^{\prime \prime}$. They correct their intensities for an extinction found from the Balmer decrement, These corrected intensities are shown in the third column of Table 8 for some of the lines of interest to us. Notice that the [Ne III] line at $\lambda 3869 \AA$ was too weak to measure. Williams et al. (2008) do not report the intensities of any of the He r lines; for the intensities of these lines the spectrum reported by Kingsburgh \& Barlow (1994) are used. These are shown in Col. 4 of the table, where the average intensity is weighted to the spectrum of Williams et al. (2008).

\subsection{The ultraviolet spectrum}

There are 14 IUE spectra of Tc 1: three high resolution shortwave spectra, seven low resolution shortwave spectra and four low resolution longwave spectra. Only a few lines are strong enough for a good identification as a nebular line however. In addition the spectrum is of low excitation so that the connection between the ultraviolet and visual spectra through the He II 
Table 9. UV spectrum of Tc 1.

\begin{tabular}{lcccc}
\hline \hline$\lambda$ & Ion & \multicolumn{3}{c}{ Intensities } \\
\cline { 3 - 5 }$(\AA)$ & & $(1)$ & $(2)$ & $(\mathrm{I} / \mathrm{H} \beta)$ \\
\hline 1906 & $\left.\mathrm{C}_{\mathrm{III}}\right]$ & 1.45 & 7.6 & 14.8 \\
1909 & $\left.\mathrm{C}_{\mathrm{III}}\right]$ & 1.16 & 6.1 & 11.9 \\
2325 & $\left.\mathrm{C}_{\mathrm{II}}\right]$ & 3.90 & 23.4 & 45.5 \\
\hline
\end{tabular}

Notes. ${ }^{(1)}$ Measured intensity from in units of $10^{-12} \mathrm{erg}^{-2} \mathrm{~s}^{-1}$. (2) Intensity corrected for extinction in units of $10^{-12} \mathrm{erg} \mathrm{cm}^{-2} \mathrm{~s}^{-1}$. I/H $\beta$ is normalized to $\mathrm{H} \beta=100$.

lines of $\lambda 1640 \AA$ and $\lambda 4686 \AA$ cannot be made. However the nebula is small enough so that almost all of its emission is measured. Feibelman (1983) has reported measuring the [O II] line at $\lambda 2471 \AA$ which can be used to connect the ultraviolet spectrum to the visual spectrum but we feel that the spectra are too noisy to measure this line. We find that only two lines are clearly measurable on the low resolution spectra. The high resolution spectra shows more lines but because they may be interstellar or stellar we do not report them here. Table 9 lists our measurements. The extinction correction is made using a value of $C=0.33$ as found by Williams et al. (2008).

\subsection{Electron density}

The ions used to determine $N_{\mathrm{e}}$ are listed in the first column of Table 4. The corresponding electron density is given in Column 7. It is about $3000 \mathrm{~cm}^{-3}$.

\subsection{Electron temperature}

Five ions have lines originating from energy levels far enough apart that their ratio is sensitive to the electron temperature. These are listed in Table 5. An electron temperature of $9000 \mathrm{~K}$ will be used with an uncertainty of less than $500 \mathrm{~K}$.

\subsection{Ionic and element abundances}

The ionic abundances have been determined using Eq. (1) above with an electron temperature of $9000 \mathrm{~K}$ and a density of $3000 \mathrm{~cm}^{-3}$. The results are given in Table 10, where the first column lists the ion concerned, the second column the line used for the abundance determination and the third column gives the intensity of the line used relative to $\mathrm{H} \beta=100$. The fourth column shows the ionic abundances, and the fifth column gives the Ionization Correction Factor (ICF), determined with the help of the model described below. In all cases when the ICF is greater than 1 , the principal ionization stage of that element has been observed.

\section{He2-108}

He2-108 (PN G316.1+08.4, also known as IRAS 14147-5156) is morphologically quite similar to NGC 1535 and Tc 1 . It is roughly circular and has a size at the $10 \%$ level of $13.6^{\prime \prime} \times$ 12.3" (Tylenda et al. 2003). A somewhat smaller diameter (11") is given by Acker et al. (1992). The size is small enough so that most of the radiation can be measured in the IUE diaphragm.

The $6 \mathrm{~cm}$ continuum radio flux density has been measured by Milne \& Aller (1975) as 33 mJy. Milne \& Aller (1982) have measured the $2 \mathrm{~cm}$ radio flux density as $43 \mathrm{mJy}$, which corresponds to a value of $49 \mathrm{mJy}$ at $6 \mathrm{~cm}$. An uncertain av-
Table 10. Ionic concentrations and chemical abundances in Tc 1.

\begin{tabular}{lccccc}
\hline \hline Ion & $\lambda$ & Int./H $\beta$ & $N_{\text {ion }} / N_{\mathrm{p}}$ & ICF & $N_{\text {el. } /} / N_{\mathrm{p}}$ \\
\hline $\mathrm{He}^{+}$ & 5875 & 9.0 & 0.060 & $?$ & $\geq 0.060$ \\
$\mathrm{C}^{+}$ & 2325 & 45.5 & $1.85(-4)$ & & \\
$\mathrm{C}^{++}$ & 1909 & 26.7 & $1.71(-4)$ & 1 & $3.6(-4)$ \\
$N^{+}$ & 6584 & 95.4 & $2.58(-5)$ & 1.4 & $3.6(-5)$ \\
$\mathrm{O}^{+}$ & 3727 & 130 & $1.87(-4)$ & & \\
$\mathrm{O}^{++}$ & 5007 & 124 & $6.8(-5)$ & 1 & $2.6(-4)$ \\
$\mathrm{Ne}^{+}$ & 12.8 & 37.5 & $6.2(-5)$ & & \\
$\mathrm{Ne}^{++}$ & 15.5 & 1.46 & $1.1(-6)$ & 1 & $6.3(-5)$ \\
$\mathrm{S}^{+}$ & 6731 & 3.50 & $1.1(-6)$ & & \\
$\mathrm{S}^{++}$ & 6312 & 0.47 & $1.7(-6)$ & & \\
$\mathrm{S}^{++}$ & 18.7 & 14.0 & $1.58(-6)$ & & \\
$\mathrm{S}^{+3}$ & 10.5 & 0.47 & $3.8(-8)$ & 1 & $2.8(-6)$ \\
$\mathrm{Ar}^{+}$ & 6.99 & 32.8 & $4.2(-6)$ & & \\
$\mathrm{Ar}^{++}$ & 8.99 & 6.5 & $7.0(-7)$ & & \\
$\mathrm{Ar}^{++}$ & 7135 & 5.75 & $7.0(-7)$ & 1 & $5.1(-6)$ \\
$\mathrm{Cl}^{++}$ & 5538 & 0.303 & $5.9(-8)$ & 1.6 & $9.4(-8)$ \\
$\mathrm{P}^{++}$ & 17.9 & 0.735 & $1.67(-7)$ & 1.2 & $2.0(-7)$ \\
$\mathrm{Fe}^{++}$ & 22.9 & 0.295 & $1.1(-7)$ & 1.4 & $1.54(-7)$ \\
\hline
\end{tabular}

Notes. Wavelength in Angstrom for all values of $\lambda$ above 1000, otherwise in $\mu \mathrm{m}$. Intensities given with respect to $\mathrm{H} \beta=100$.

erage value of $39 \mathrm{mJy}$ at $6 \mathrm{~cm}$ corresponds to an $\mathrm{H} \beta$ flux of $1.26 \times 10^{-11} \mathrm{erg} \mathrm{cm}^{-2} \mathrm{~s}^{-1}$. The measured $\mathrm{H} \beta$ flux is $3.7 \times$ $10^{-12} \mathrm{erg} \mathrm{cm}^{-2} \mathrm{~s}^{-1}$ (see Acker et al. 1992) which leads to an extinction coefficient $C=0.53$.

\subsection{Infrared spectrum}

The IRS measurement of He2-108 was centered at RA(2000) $14^{\mathrm{h}} 18^{\mathrm{m}} 08.4^{\mathrm{s}}$ and $\operatorname{Dec}(2000)-52^{\circ} 10^{\prime} 38.0^{\prime \prime}$. This is a slight mispointing from the center measured by Kerber et al. (2003) of $\operatorname{RA}(2000) 14^{\mathrm{h}} 18^{\mathrm{m}} 08.89^{\mathrm{s}}$ and $\operatorname{Dec}(2000)-52^{\circ} 10^{\prime} 39.7^{\prime \prime}$, which is presumably the coordinate of the central star. This does not have an important effect for the LH measurement because of the large $\mathrm{LH}$ diaphragm. It does however affect the SH measurement for which the Nod 1 measurement measured only part of the nebula. The Nod 2 measurement fell within the nebula so that we have only used the Nod 2 measurement. By equating the continuum measured at $19 \mu \mathrm{m}$ in the LH measurement with the same continuum measured in the $\mathrm{SH}$ Nod 2 we obtain a ratio of $\mathrm{LH} / \mathrm{SH}=2.5$. The ratio of $\mathrm{SH}$ to $\mathrm{SL}$ was obtained by equating the [Ne II] fluxes in the two measurements. All fluxes were measured using the Gaussian line-fitting routine. The measured emission line intensities are given in Table 1, after correcting the SH measurements by the factor 2.5 and the SL measurements by a factor of 4.07 . The $\mathrm{H} \beta$ flux found from the infrared hydrogen lines (especially the lines at $7.48 \mu \mathrm{m}$ and $12.37 \mu \mathrm{m}$ ) using the theoretical ratios of Hummer \& Storey (1987), is $1.15 \times 10^{-11} \mathrm{erg} \mathrm{cm}^{-2} \mathrm{~s}^{-1}$, which is only slighly smaller than the $\mathrm{H} \beta$ found from the radio flux density. This indicates that the LH diaphragm measured almost the entire nebula.

\subsection{Visual spectrum}

There are two measurements of the visual spectrum of He2108 , probably because it is weak and not visible to northern observatories. The measurements by Torres-Peimbert \& Peimbert (1977) are considered by these authors to be less accurate than measurements of other PNe they have made. An accuracy of about $30 \%$ is given by these authors. The visual spectrum has also been measured in the 1990 Acker-Stenholm ESO survey of southern PNe. The full results of this survey have not yet been 
Table 11. Visual spectrum of He2-108.

\begin{tabular}{|c|c|c|c|c|}
\hline \multirow{2}{*}{$\begin{array}{l}\lambda \\
(\AA)\end{array}$} & \multirow[t]{2}{*}{ Ion } & \multicolumn{2}{|c|}{ Intensities $^{(\dagger)}$} & \multirow{2}{*}{$\begin{array}{l}\text { Average } \\
\text { Intens. }\end{array}$} \\
\hline & & (1) & (2) & \\
\hline 3727 & {$\left[\mathrm{O}_{\mathrm{II}}\right]$} & 138 & & 138 \\
\hline 3869 & [Ne III] & 4.7 & 20: & 5: \\
\hline 4340 & $\mathrm{H} \gamma$ & 43 & 44 & 43 \\
\hline 4363 & [O III] & 1.1 & & 1.1: \\
\hline 4471 & $\mathrm{He}_{\mathrm{I}}$ & 6.8 & & 6.8 \\
\hline 4861 & $\mathrm{H} \beta$ & 100 & 100 & 100 \\
\hline 5007 & [O III] & 200 & 167 & 190 \\
\hline 5876 & $\mathrm{He}_{\mathrm{I}}$ & 17 & 17.5 & 17.5 \\
\hline 6563 & $\mathrm{H} \alpha$ & 295 & 310 & 300 \\
\hline 6584 & [N II] & 105 & 90 & 100 \\
\hline 7135 & [Ar III] & & 15 & 15 \\
\hline $\mathrm{C}(\mathrm{H} \beta)$ & & 0.4 & 0.5 & \\
\hline
\end{tabular}

Notes. ${ }^{(\dagger)}$ References: (1) Torres-Peimbert \& Peimbert (1977); (2) Acker (priv. comm.). ${ }^{(:)}$Indicates uncertain values. ${ }^{(C)}$ Is the extinction (see text)

published, but Acker has sent us the reduced spectrum of this object. The results are given in Table 11. The values are corrected for extinction; those in Col. 3 by the authors and those in Col. 4 by us in an attempt to produce the expected Balmer decrement. The differences in the blue part of the spectrum reflects uncertainty of the measurements.

\subsection{The ultraviolet spectrum}

There are 6 IUE spectra of He2-108: 4 low resolution shortwave spectra and 2 low resolution longwave spectra. The spectra are dominated by the bright central star. Our interest was to look for evidence for nebular emission in the carbon ions, either [C III] $\lambda 1907 \AA$ or [ $\left.\mathrm{C}_{\text {II }}\right] \lambda 2325 \AA$. No [C II] emission could be seen. A [C III] $\lambda 1907 \AA$ line is seen in one of the four shortwave spectra: SWP14181. Strangely this line is not seen in the other three spectra whose exposure time is longer than SWP14181. The observed line is well above the noise and is at the correct wavelength, further the position of all four spectra are the same and only the position angle is different. Until new observations are available we will regard this measurement as an upper limit to the intensity. The observed value is $3 \times 10^{-13} \mathrm{erg} \mathrm{cm}^{-2} \mathrm{~s}^{-1}$, which after correction for extinction becomes $3.3 \times 10^{-12} \mathrm{erg} \mathrm{cm}^{-2} \mathrm{~s}^{-1}$. There are no other obvious nebular lines in these spectra.

\subsection{Electron density}

There is only a single ion where a line ratio can be used to determine $N_{\mathrm{e}}$. This is the [S III] ratio 33.4/18.7 in the infrared. The ratio measured, 0.485 , leads to a density of $2200 \mathrm{~cm}^{-3}$. The ratio of the [Ar III] lines in the infrared, 8.99/21.8, is not very dependent on the density in this range but it is consistent with this value of density. The abundances are not dependent on the density in this range.

\subsection{Electron temperature}

Only three ions have lines originating from energy levels far enough apart that their ratio is sensitive to the electron temperature. These are listed in Table 5. The temperature found from the [O III] lines is uncertain. A somewhat lower value of 8730 is given by McKenna et al. (1996) but the authors do not give the
Table 12. Ionic concentrations and chemical abundances in He2-108.

\begin{tabular}{lccccc}
\hline \hline Ion & $\lambda$ & Int./H $\beta$ & $N_{\text {ion }} / N_{\mathrm{p}}$ & ICF & $N_{\text {el. }} / N_{\mathrm{p}}$ \\
\hline $\mathrm{He}^{+}$ & 5875 & 17 & 0.11 & & 0.11 \\
$\mathrm{C}^{++}$ & 1909 & $\leq 18$ & $\leq 1.7(-4)$ & 1.1 & $\leq 1.9(-4)$ \\
$N^{+}$ & 6584 & 105 & $3.2(-5)$ & 1.9 & $6.0(-5)$ \\
$\mathrm{O}^{+}$ & 3727 & 67 & $1.4(-4)$ & & \\
$\mathrm{O}^{++}$ & 5007 & 200 & $1.35(-5)$ & 1 & $2.8(-4)$ \\
$\mathrm{Ne}^{+}$ & 12.8 & 102 & $1.72(-4)$ & & \\
$\mathrm{Ne}^{++}$ & 15.5 & 16.5 & $1.14(-5)$ & 1 & $2.9(-4)$ \\
$\mathrm{S}^{+}$ & 6731 & 2 & $1.4(-7)$ & & \\
$\mathrm{S}^{++}$ & 18.7 & 72.2 & $7.46(-6)$ & & \\
$\mathrm{S}^{+3}$ & 10.5 & 3.6 & $4.4(-7)$ & 1 & $8.1(-6)$ \\
$\mathrm{Ar}^{++}$ & 8.99 & 25 & $2.7(-6)$ & 1.9 & $5.1(-6)$ \\
$\mathrm{Fe}^{++}$ & 22.9 & 1.02 & $3.6(-7)$ & 1.2 & $4.0(-7)$ \\
\hline
\end{tabular}

Notes. Wavelength in Angstrom for all values of $\lambda$ above 1000, otherwise in $\mu \mathrm{m}$. Intensities given with respect to $\mathrm{H} \beta=100$.

details of the spectra. We will use a temperature of $8500 \mathrm{~K}$ with an uncertainty of about $500 \mathrm{~K}$.

\subsection{Ionic and element abundances}

The ionic abundances have been determined using Eq. (1) above with an electron temperature of $8500 \mathrm{~K}$ and a density of $2200 \mathrm{~cm}^{-3}$. The results are given in Table 12, where the columns are arranged as in Table 12. The ICF is usually 1, except for nitrogen where it is assumed that the ratio $N^{+} / N^{++}=\mathrm{O}^{+} / \mathrm{O}^{++}$. The principal ionization stage has been measured in iron, argon and carbon but a small correction has been made for the singly ionized state. For argon, the similarity of the ionization potentials to nitrogen is the basis for the ICF used.

\section{NGC 6629}

NGC 6629 (PN G009.4-5.0, IRAS 18226-2313) is classified as an elliptical, almost round nebula. It is slightly larger than Tc1 and He2-108, but smaller than NGC 1535. Its size down to the $10 \%$ level is given by Tylenda et al. (2003) as $16.6^{\prime \prime} \times 15.5^{\prime \prime}$. It is surrounded by a halo which has a diameter of about $40^{\prime \prime}$ and is more compressed on the north side. The $6 \mathrm{~cm}$ radio flux density is given by Griffith et al. (1994) as $277 \mathrm{mJy}$. Milne \& Aller (1975) give a $6 \mathrm{~cm}$ flux density of $292 \mathrm{mJy}$ while Milne \& Aller (1982) find a $2 \mathrm{~cm}$ flux density of $234 \mathrm{mJy}$, which corresponds to a value of $260 \mathrm{mJy}$ at $6 \mathrm{~cm}$. We will use a value of $270 \mathrm{mJy}$ for the $6 \mathrm{~cm}$ radio flux density which predicts a value of $\mathrm{H} \beta=9.3 \times 10^{-11} \mathrm{erg} \mathrm{cm}-2 \mathrm{~s}^{-1}$ for the values of electron temperature and helium abundance given below. Since the observed $\mathrm{H} \beta=1.18 \times 10^{-11} \mathrm{erg} \mathrm{cm}^{-2} \mathrm{~s}^{-1}$ the extinction constant $C=0.896$ or $E_{B-V}=0.61$.

\subsection{Infrared spectrum}

The IRS measurement of NGC 6629 was centered at RA(2000) $18^{\mathrm{h}} 25^{\mathrm{m}} 42.5^{\mathrm{s}}$ and $\operatorname{Dec}(2000)-23^{\circ} 12^{\prime} 10.1^{\prime \prime}$. This is very close to the center measured by Kerber et al. (2003) of RA(2000) $18^{\mathrm{h}} 25^{\mathrm{m}} 42.45^{\mathrm{s}}$ and $\operatorname{Dec}(2000)-23^{\circ} 12^{\prime} 10.59^{\prime \prime}$, which is presumably the coordinate of the central star. Because the nebula has a size close to the size of the large LH diaphragm most of the nebula was within the LH diaphragm. The SH diaphragm measured only part of the nebula. By equating the continuum measured at $19 \mu \mathrm{m}$ in the LH measurement with the same continuum measured in the $\mathrm{SH}$ diaphragm we obtain a ratio of $\mathrm{LH} / \mathrm{SH}=2.7$. 
Table 13. Visual spectrum of NGC 6629.

\begin{tabular}{|c|c|c|c|c|}
\hline \multirow{2}{*}{$\begin{array}{l}\lambda \\
(\AA)\end{array}$} & \multirow[t]{2}{*}{ Ion } & \multicolumn{2}{|c|}{ Intensities ${ }^{\dagger}$} & \multirow{2}{*}{$\begin{array}{l}\text { Averag } \\
\text { Intens. }\end{array}$} \\
\hline & & (1) & (2) & \\
\hline 3727 & {$\left[\mathrm{O}_{\mathrm{II}}\right]$} & 36.2 & 41.1 & 38 \\
\hline 3869 & [Ne III] & 40.8 & 33.3 & 37 \\
\hline 4340 & $\mathrm{H} \gamma$ & 46.3 & 46.5 & 46.4 \\
\hline 4363 & {$\left[\mathrm{O}_{\mathrm{III}}\right]$} & 2.7 & 2.8 & 2.75 \\
\hline 4471 & $\mathrm{He}_{\mathrm{I}}$ & 4.6 & 4.43 & 4.5 \\
\hline 4861 & $\mathrm{H} \beta$ & 100 & 100 & 100 \\
\hline 5007 & [O III] & 670 & 674 & 672 \\
\hline 5517 & {$\left[\mathrm{Cl}_{\text {III }}\right]$} & 0.45 & & 0.45 \\
\hline 5538 & {$\left[\mathrm{Cl}_{\mathrm{III}}\right]$} & 0.4 & & 0.4 \\
\hline 5876 & $\mathrm{He}_{\mathrm{I}}$ & 15.2 & 12.0 & 14 \\
\hline 6312 & [S III] & 0.5 & & 0.5 \\
\hline 6563 & $\mathrm{H} \alpha$ & 286 & 289 & 287 \\
\hline 6584 & {$\left[\mathrm{~N}_{\text {III }}\right]$} & 10.8 & 11.0 & 10.9 \\
\hline 6717 & {$\left[\mathrm{~S}_{\mathrm{II}}\right]$} & 0.6 & 0.53 & 0.53 \\
\hline 6731 & {$\left[\mathrm{~S}_{\text {II }}\right]$} & 0.7 & 0.71 & 0.71 \\
\hline 7135 & [Ar III] & 12.7 & 12.1 & 12.4 \\
\hline 9532 & {$\left[\mathrm{~S}_{\mathrm{III}}\right]$} & 29.2 & & 29.2 \\
\hline $\mathrm{C}(\mathrm{H} \beta)$ & & 0.8 & 0.96 & \\
\hline
\end{tabular}

Notes. ${ }^{(\dagger)}$ References; (1) Milingo et al. (2002a); (2) Aller \& Keyes. (1987). (:) Indicates uncertain values. ${ }^{(C)}$ Is the extinction (see text).

This number is somewhat uncertain because the spectrum is rather noisy. This ratio can also be obtained by comparing both the [Ne III] 15.5/36.0 line ratio and the [Ar III] 21.8/8.99 line ratio since both of these ratios have only a small dependence in electron density and temperature. We obtain an $\mathrm{LH} / \mathrm{SH}$ ratio of 1.71 from the [Ne III] lines and a value of 2.44 from the [Ar III] lines. An average value of $\mathrm{LH} / \mathrm{SH}=2.1$ was used. The ratio of $\mathrm{SH}$ to SL was obtained by equating the $[\mathrm{Ne}$ II] the [S IV] fluxes in the two measurements which leads to $\mathrm{SH} / \mathrm{SL}=1.10$. All fluxes were measured using the Gaussian line-fitting routine.The measured emission line intensities are given in Table 1, after correcting the SH measurements by the factor 2.1 and the SL measurements by a factor of 2.3. The $\mathrm{H} \beta$ flux found from the infrared hydrogen lines (especially the lines at $7.48 \mu \mathrm{m}$ and $12.37 \mu \mathrm{m}$ ) using the theoretical ratios of Hummer \& Storey (1987) at a temperature of $8600 \mathrm{~K}$ is $8.45 \times 10^{-11} \mathrm{erg} \mathrm{cm}^{-2} \mathrm{~s}^{-1}$, which is only slighly smaller than the $\mathrm{H} \beta$ found from the radio flux density. This indicates that the LH diaphragm measured most of the nebula.

\subsection{Visual spectrum}

There are several measurements of the visual spectrum of NGC 6629 in the literature. The two measurements quoted here are probably the best. These are those of Milingo et al. (2002a) and Aller \& Keyes (1987) and are given in Table 13. In addition Kingsburgh \& English (1992) have measured the [O II] ratio $3726 / 3729$ to be $1.63 \pm 0.50$ and the [S II] ratio $6717 / 6731$ to be $0.74 \pm 0.04$.

The extinction coeficients, $C$, found from the Balmer decrement and given at the bottom of the table, are essentially the same as that found from the radio flux density (given at the beginning of this section).

\subsection{The ultraviolet spectrum}

There are seven IUE spectra of NGC 6629: three low resolution shortwave spectra and four low resolution longwave spectra. The diaphragm was centered near the edge of the nebula for some
Table 14. Ionic concentrations and chemical abundances in NGC 6629.

\begin{tabular}{lccccc}
\hline \hline Ion & $\lambda$ & Int./H $\beta$ & $N_{\text {ion }} / N_{\mathrm{p}}$ & ICF & $N_{\text {el } /} / N_{\mathrm{p}}$ \\
\hline $\mathrm{He}^{+}$ & 5875 & 14.0 & 0.094 & $?$ & 0.096 \\
$\mathrm{C}^{++}$ & 1909 & 23.7 & $1.9(-4)$ & 1.1 & $2.1(-4)$ \\
$N^{+}$ & 6584 & 10.9 & $3.23(-6)$ & 14 & $4.5(-5)$ \\
$\mathrm{O}^{+}$ & 3727 & 38 & $3.5(-5)$ & & \\
$\mathrm{O}^{++}$ & 5007 & 672 & $4.4(-4)$ & 1.1 & $4.8(-4)$ \\
$\mathrm{Ne}^{+}$ & 12.8 & 9.95 & $1.8(-5)$ & & \\
$\mathrm{Ne}^{++}$ & 15.5 & 89.1 & $6.06(-5)$ & & \\
$\mathrm{Ne}^{++}$ & 3869 & 37 & $7.2(-5)$ & 1 & $8.4(-5)$ \\
$\mathrm{S}^{+}$ & 6731 & 0.7 & $5.3(-8)$ & & \\
$\mathrm{S}^{++}$ & 18.7 & 18.2 & $1.77(-6)$ & & \\
$\mathrm{S}^{+3}$ & 10.5 & 14.4 & $3.9(-7)$ & 1 & $2.2(-6)$ \\
$\mathrm{Ar}^{++}$ & 8.99 & 12.7 & $1.35(-6)$ & & \\
$\mathrm{Ar}^{++}$ & 7135 & 12.4 & $1.67(-7)$ & 1.2 & $2(-6)$ \\
$\mathrm{Cl}^{++}$ & 5538 & 0.4 & $9.7(-8)$ & $1.2:$ & $1.2(-8):$ \\
$\mathrm{P}^{++}$ & 17.9 & 0.61 & $4.5(-8)$ & $1.2:$ & $2.0(-7):$ \\
\hline
\end{tabular}

Notes. Wavelength in Angstrom for all values of $\lambda$ above 1000, otherwise in $\mu \mathrm{m}$. Intensities given with respect to $\mathrm{H} \beta=100$.

of the spectra and closer to the central star for other spectra. All spectra appear to be dominated by the bright central star. This indicates that much of the nebula is within the diaphragm but it is difficult to specify exactly how much of the nebula is being measured. The only clearly nebular emission is the [C III] $\lambda 1907 \AA$ line which is seen in all three shortwave spectra. It has the same intensity in all spectra: $2.5 \times 10^{-13} \mathrm{erg} \mathrm{cm}^{-2} \mathrm{~s}^{-1}$. Corrected for extinction this becomes $2.2 \times 10^{-11} \mathrm{erg} \mathrm{cm}^{-2} \mathrm{~s}^{-1}$; thus the ratio of the line to $\mathrm{H} \beta$ is 23.7 (when $\mathrm{H} \beta=100$ ). This value will be used when determining the carbon abundance but it is a lower limit since some of the nebular emission may be outside the nebula. Possible [C II] $\lambda 2325 \AA$ A cannot be seen.

\subsection{Electron density}

The ions used to determine $N_{\mathrm{e}}$ are listed Table 4 . The electron density appears to be about $2000 \mathrm{~cm}^{-3}$.

\subsection{Electron temperature}

Four ions have lines originating from energy levels far enough apart that their ratio is sensitive to the electron temperature. These are listed in Table 5. An electron temperature of $8700 \mathrm{~K}$ is found with an uncertainty of less than $300 \mathrm{~K}$. No temperature gradient is apparent.

\subsection{Ionic and element abundances}

The ionic abundances have been determined using Eq. (1) above with an electron temperature of $8700 \mathrm{~K}$ and a density of $2000 \mathrm{~cm}^{-3}$. The results are given in Table 14, where, as in Table 6, the first column lists the ion concerned, the second column the line used for the abundance determination and the third column gives the intensity of the line used relative to $\mathrm{H} \beta=100$. The fourth column shows the ionic abundances, and the fifth column gives the Ionization Correction Factor (ICF), determined empirically. In all cases but one, when the ICF is greater than 1 , the principal ionization stage of that element has been observed. The single exception is nitrogen, where it is assumed that $N^{+} / N^{++}=\mathrm{O}^{+} / \mathrm{O}^{++}$. 


\subsection{Errors}

We refer here to possible abundance errors in all of the PNe studied here. This is difficult to specify because there are errors due to the measurements, the electron temperature and the ICF. There is only a neglible error due to uncertainties in the electron density. The measurement error depends on the strength of the line; for the stronger lines it is probably less than $15 \%$. The values of $T_{\mathrm{e}}$ appear to be independent of the ionization potential in all PNe considered here. For at least two of the nebulae the uncertainty could be as large as $1000 \mathrm{~K}$. The temperature uncertainty plays only a small role for the infrared lines but is much more important for the ultraviolet lines. Taken together we estimate that for all elements except carbon the abundance uncertainties are not more than $20-30 \%$ for those elements for the ICF is close to unity. When the ICF is higher than 1.5 the abundance uncertainties are about $50 \%$. For carbon the ICF is usually unity but the abundance is very temperature dependent. The error is probably slightly higher, of the order of $50 \%$ for this element. The largest error for helium occurs in the PNe with low temperature central stars where neutral helium is present. This occurs in Tc1, but may also occur in NGC 6629 and He2-108. Other sources of error for the helium abundance are probably small.

\section{Model}

In order to obtain as nearly a correct model as possible, the star as well as the nebula must be considered. Modeling the nebulastar complex will allow characterizing not only the central star's temperature but other stellar parameters as well (i.e., $\log g$ and luminosity). It can determine distance and other nebular properties, especially the composition, including the composition of elements that are represented by a single stage of ionization, which cannot be determined by the simplified analysis above. This method can take the presence of dust and molecules into account in the nebular material, when there is any there, making it a very comprehensive approach. While the line ratio method is simple and fast, the ICFs rest on uncertain physics. To this end, modeling serves as an effective means, and the whole set of parameters are determined in a unified way, assuring self consistency. Also, in this way one gets good physical insight into the PN, the method and the observations. Thus, modeling is a good approach to an end-to-end solution to the problem. We used Cloudy version C08.00 for this work.

\section{1. $T c 1$}

\subsubsection{Assumptions}

Tylenda et al. (2003) give a diameter of $12.9^{\prime \prime} \times 12.2^{\prime \prime}$ for this $\mathrm{PN}$. We have used a diameter of $12.55^{\prime \prime}$ in our modeling.

\subsubsection{Model results}

Numerous models were run and we found that there was the primary problem of fixing the stellar effective temperature. While the ionization of carbon, neon and argon indicated a somewhat lower $T_{\text {eff }}$, the observed [O III] lines required a higher value. We tried a range of temperatures, distances, densities, density profiles and various model atmospheres. Some models have been tried with stellar wind and some without while some with simple black body atmospheres for the CSPN. Observed [O III] line fluxes seem to be unusually high and in trying to match them, $\mathrm{Ne}$
Table 15. Parameters representing the final model for Tc1.

\begin{tabular}{|c|c|c|c|c|}
\hline Parameter & \multicolumn{4}{|c|}{ Value } \\
\hline Ionizing source & \\
\hline Model atmosphere & \multicolumn{4}{|c|}{ WMbasic } \\
\hline$T_{\text {eff }}$ & \multicolumn{4}{|c|}{$34700 \mathrm{~K}$} \\
\hline $\log g$ & \multicolumn{4}{|c|}{3.30} \\
\hline $\log z$ & \multicolumn{4}{|c|}{-0.3} \\
\hline Luminosity & \multicolumn{4}{|c|}{$1480 L_{\odot}$} \\
\hline \multicolumn{5}{|l|}{ Nebula } \\
\hline Density profile & \multicolumn{4}{|c|}{ constant density $2850 / \mathrm{cc}$} \\
\hline \multirow[t]{6}{*}{ Abundance } & $\mathrm{H}$ & $\mathrm{He}$ & $\mathrm{C}$ & $\mathrm{N}$ \\
\hline & 12.000 & 10.91 & 8.674 & 7.590 \\
\hline & $\mathrm{O}$ & $\mathrm{Ne}$ & $\mathrm{Mg}$ & $\mathrm{Si}$ \\
\hline & 8.431 & 7.4 & $5.5^{\circ}$ & 5.778 \\
\hline & $\mathrm{P}$ & S & $\mathrm{Cl}$ & $\mathrm{Ar}$ \\
\hline & 5.3 & 6.203 & 4.963 & 6.478 \\
\hline Size & \multicolumn{4}{|c|}{$6.275^{\prime \prime}$ (radius) } \\
\hline Distance & \multicolumn{4}{|c|}{$1.80 \mathrm{kpc}$} \\
\hline Dust grains & \multirow{2}{*}{\multicolumn{4}{|c|}{$\begin{array}{c}\text { Graphites of single size } 1.0 \mu \mathrm{m} ; \\
1.077 \mathrm{e} 16 \mathrm{~cm}\end{array}$}} \\
\hline inner radius & & & & \\
\hline outer radius & \multicolumn{4}{|c|}{$1.690 \mathrm{e} 17 \mathrm{~cm}$} \\
\hline Filling factor & \multicolumn{4}{|c|}{1.0} \\
\hline
\end{tabular}

and Ar moved up to higher stages of ionization. Many observed lines would suggest a cooler $T_{\text {eff }}$ than what our final model indicated. In the final model, shown in Table 15, we have used a low metallicity model atmosphere mainly to take care of infusing more photons in the wavelength region below $912 \AA$ without increasing $T_{\text {eff }}$. The metallicity is only half that of Sun. The value of gravity was also kept as low as possible for a similar boost in the input stellar radiation. We note that the galactic latitude of the CSPN is only around -9 degrees and the fact that we were forced to use a low metallicity model atmosphere for such a low latitude object shows the extreme to which a modeler is driven, when faced with anomalous nebular line emission.

At this stage we were not sure whether any extra source of energy was present, as there was no observational clue, but the above facts forced us to look at all different possibilities. It might as well be that model atmospheres do not realistically represent the stellar radiation. Another fact is that the accuracy of the optical spectra containing the $\mathrm{O}$ III lines is quite high, an error of only $8 \%$ is quoted by the authors for the spectrophotometry. Therefore we surmise that something very interesting is happening in the formation of $\mathrm{O}$ III lines but are unable to hazard any guess. But while we did these adjustments in modeling, lines of neon, argon and sulphur gave trouble. The sulphur lines were improved to get a better fit by adjusting the DR (dielectronic recombination) rates since the DR rates are poorly known. The final model output spectra are presented in Table 16, where it is clear that the model fluxes for most low ionization stages (O II, $\mathrm{C}$ II, and Ne II) are too high.

To reproduce the observed IR dust continua, we found that graphites gave a better fit and used them in our modeling rather than silicates. The grains included in the modeling were the Cloudy's set called Orion distribution which has a bias towards larger grain sizes. The match to the observed IR continua is reasonable (see Fig. 1). So the final model we present is the best we could achieve. Overall we feel our exercise raises more pertinent questions than answers as this PN seems to throw lots of challenges to our current understanding of nebular physics. We are inclined to recommend the abundances as determined by the ICF method for this PN. A very pertinent point we want to highlight 
Table 16. The emission line fluxes $(\mathrm{H} \beta=100)$ for $\mathrm{Tc} 1$.

\begin{tabular}{|c|c|c|c|c|c|c|c|}
\hline Label & Line $^{\dagger}$ & Model flux & $\begin{array}{l}\text { Obsd. flux } \\
\text { (dereddened) }\end{array}$ & Label & Line & Model flux & $\begin{array}{l}\text { Obsd. flux } \\
\text { (dereddened) }\end{array}$ \\
\hline TOTL & $4861 \mathrm{~A}$ & 100.00 & 100.00 & S 3 & $6312 \mathrm{~A}$ & 0.40 & 0.46 \\
\hline C 2 & $1335 \mathrm{~A}$ & 10.35 & 11.43 & O 1 & $6363 \mathrm{~A}$ & 0.78 & 0.05 \\
\hline C 3 & $1478 \mathrm{~A}$ & 0.00 & 6.65 & $\mathrm{~N} 2$ & $6548 \mathrm{~A}$ & 32.43 & 30.10 \\
\hline C 1 & $1561 \mathrm{~A}$ & 0.34 & 1.26 & $\mathrm{~N} 2$ & $6584 \mathrm{~A}$ & 95.70 & 93.20 \\
\hline C 2 & $1761 \mathrm{~A}$ & 0.18 & 2.08 & S II & $6716 \mathrm{~A}$ & 2.38 & 2.15 \\
\hline C 3 & 1907A & 16.56 & 14.76 & S II & $6731 \mathrm{~A}$ & 3.70 & 3.41 \\
\hline C 3 & $1910 \mathrm{~A}$ & 11.85 & 11.94 & Ar 3 & $7135 \mathrm{~A}$ & 15.52 & 6.99 \\
\hline TOTL & $2326 \mathrm{~A}$ & 86.80 & 44.59 & O II & 7323A & 9.04 & 5.43 \\
\hline O II & $3726 \mathrm{~A}$ & 171.42 & 131.10 & O II & $7332 \mathrm{~A}$ & 7.23 & 4.57 \\
\hline O II & 3729A & 91.40 & 86.59 & Ar 3 & 7751A & 3.74 & 1.66 \\
\hline S II & 4070A & 0.84 & 0.62 & $\mathrm{Fe} 2$ & 8617A & 0.02 & 0.01 \\
\hline S II & 4078A & 0.27 & 0.18 & C 1 & $8727 \mathrm{~A}$ & 0.05 & 0.01 \\
\hline $\mathrm{Fe} 2$ & $4244 \mathrm{~A}$ & 0.00 & 0.02 & S 3 & 9069A & 7.64 & 12.51 \\
\hline $\mathrm{Fe} 2$ & $4359 \mathrm{~A}$ & 0.00 & 0.01 & TOTL & $9850 \mathrm{~A}$ & 0.54 & 0.04 \\
\hline TOTL & $4363 \mathrm{~A}$ & 0.53 & 0.56 & Ar 2 & $6.980 \mathrm{~m}$ & 14.12 & 32.84 \\
\hline P 2 & 4669A & 0.02 & 0.01 & Ar 3 & $9.000 \mathrm{~m}$ & 16.23 & 6.49 \\
\hline O 3 & 4959A & 40.50 & 41.71 & S 4 & $10.51 \mathrm{~m}$ & 0.51 & 0.47 \\
\hline O 3 & $5007 \mathrm{~A}$ & 121.92 & 123.00 & $\mathrm{Ne} 2$ & $12.81 \mathrm{~m}$ & 19.68 & 37.41 \\
\hline Ar 3 & $5192 \mathrm{~A}$ & 0.08 & 0.03 & $\mathrm{Ne} 3$ & $15.55 \mathrm{~m}$ & 3.24 & 1.46 \\
\hline N 1 & $5198 \mathrm{~A}$ & 0.16 & 0.02 & P 3 & $17.89 \mathrm{~m}$ & 0.72 & 0.73 \\
\hline N 1 & $5200 \mathrm{~A}$ & 0.06 & 0.02 & S 3 & $18.67 \mathrm{~m}$ & 12.47 & 14.03 \\
\hline $\mathrm{Cl} 3$ & $5518 \mathrm{~A}$ & 0.27 & 0.28 & Ar 3 & $21.83 \mathrm{~m}$ & 1.05 & 0.43 \\
\hline $\mathrm{Cl} 3$ & $5538 \mathrm{~A}$ & 0.29 & 0.30 & $\mathrm{Fe} 3$ & $22.92 \mathrm{~m}$ & 0.27 & 0.29 \\
\hline O 1 & 5577A & 0.04 & 0.00 & S 3 & $33.47 \mathrm{~m}$ & 4.77 & 6.21 \\
\hline N 2 & $5755 \mathrm{~A}$ & 1.23 & 1.08 & Si 2 & $34.81 \mathrm{~m}$ & 0.32 & 0.30 \\
\hline O 1 & $6300 \mathrm{~A}$ & 2.45 & 0.12 & & & & \\
\hline
\end{tabular}

Notes. Absolute H $\beta$ flux model: $5.83 \times 10^{-11} \mathrm{erg} \mathrm{cm}^{-2} \mathrm{~s}^{-1}$ Obsn: $6.15 \times 10^{-11} \mathrm{ergs} \mathrm{cm}^{-2} \mathrm{~s}^{-1}$. "A" in Col. "Line" signifies Angstrom; " $\mathrm{m}$ " signifies $\mu \mathrm{m}$. In col. "Label", we have followed the notation used by Cloudy for atoms and ions; this will make identifying a line in Cloudy's huge line list easy. Neutral state is indicated by " 1 " and singly ionized state by " 2 " etc., "TOTL" typically means the sum of all the lines in the doublet/multiplet; or it could mean sum of all processes: recombination, collisional excitation, and charge transfer. Some elements are represented by usual notation as per Cloudy.

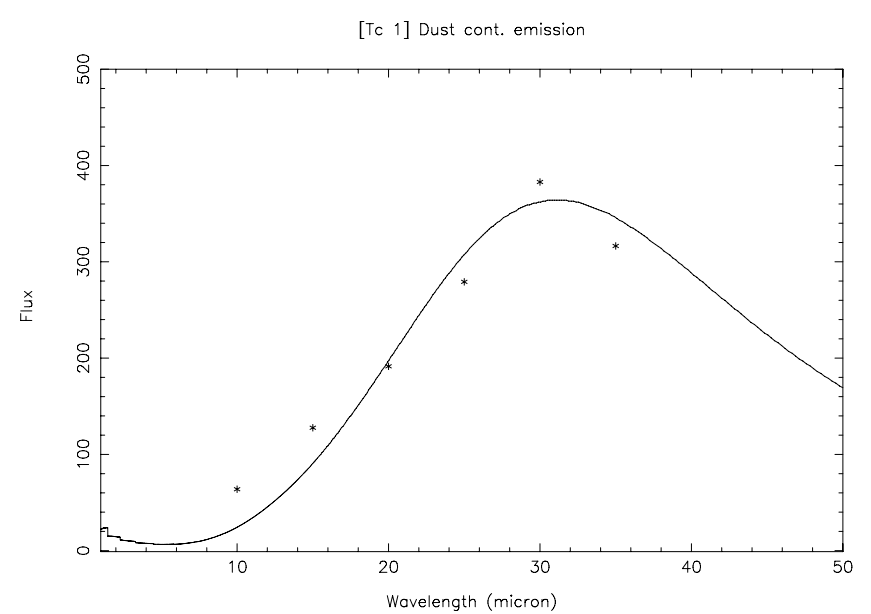

Fig. 1. The IR dust continua of Tc1. The asterisks represent the observation from Spitzer and the continuous curve is the model output.

is the fact that this PN shows nebular absorption lines too; see Williams et al. (2008) and to the best of our knowledge existing photoionization codes are yet to have a provision for computing the equivalent widths of such lines so that they can also be compared with observed values. This would make the criteria for a good fit (with observation) more stringent. Presently all models published till date used only nebular emission lines alone. Incorporating the formation of absorption lines would be the next major paradigm shift in photoionization modeling.

\subsection{Modeling NGC 1535}

We now describe our unsuccessful efforts to make a photoionization model for this PN. It is necessary to go into the details since it reveals insights into aspects of nebular physics which are normally taken for granted as well known.

\subsubsection{Assumptions}

The appearance of NGC 1535 from an image taken by Schwarz et al. (1992) is nearly spherical. The IR and radio measurements described earlier give an absolute $\mathrm{H}_{\beta}$ flux that is consistent with a diameter of $45^{\prime \prime}$, that includes a low density outer zone beyond a high density innerzone of diameter of $19^{\prime \prime}$. We wanted to include a density profile for the nebula to describe the variation of the number density $\mathrm{N}(\mathrm{H})$ with the radius and so derived a template profile from the $\mathrm{H}_{\alpha}$ image taken from http: //astro. uni-tuebingen.de/groups/pn/. The image was taken on an as-is basis and imported into the IRAF and using cross-cuts at different azimuths an average profile was generated and then normalized to have a peak value of $1300 / \mathrm{cc}$. The nebular radius was normalized to $22.5^{\prime \prime}$, to include the low density region as well. We used this profile as a starting value but later have experimented with modifications to it as well as tried simple constant density models. Though the presence of dust and $\mathrm{H}_{2}$ molecules is observed in this PN we did not include them in our simulations. 
A\&A 531, A23 (2011)

Table 17. Elemental abundance of PNe with far-infrared data in addition to optical and UV data.

\begin{tabular}{lcccccccccc}
\hline \hline PNe & $\mathrm{He} / \mathrm{H}$ & $\begin{array}{c}\mathrm{C} / \mathrm{H} \\
\times 10^{-4}\end{array}$ & $\begin{array}{c}\mathrm{N} / \mathrm{H} \\
\times 10^{-4}\end{array}$ & $\begin{array}{c}\mathrm{O} / \mathrm{H} \\
\times 10^{-4}\end{array}$ & $\begin{array}{c}\mathrm{Ne} / \mathrm{H} \\
\times 10^{-4}\end{array}$ & $\begin{array}{c}\mathrm{S} / \mathrm{H} \\
\times 10^{-6}\end{array}$ & $\begin{array}{c}\mathrm{Ar} / \mathrm{H} \\
\times 10^{-6}\end{array}$ & $\begin{array}{c}\mathrm{Cl} / \mathrm{H} \\
\times 10^{-8}\end{array}$ & $\begin{array}{c}\mathrm{R}^{\natural} \\
(\mathrm{kpc})\end{array}$ & Ref. \\
\hline NGC 1535 & 0.091 & 1.6 & 0.32 & 2.7 & 0.54 & $! .3$ & 1.1 & 6.0 & 9.9 & $\mathrm{a}$ \\
Tc 1 & $\geq 0.060$ & 3.6 & 0.36 & 2.6 & 0.63 & 2.8 & 5.1 & 9.4 & 6.3 & $\mathrm{a}$ \\
He2-108 & 0.11 & $\leq 1.9$ & 0.60 & 2.8 & 2.9 & 8.1 & 5.1 & & 4.1 & $\mathrm{a}$ \\
NGC 6629 & 0.096 & 2.1 & 0.45 & 4.8 & 0.84 & 2.2 & 2.0 & 12. & 6.0 & $\mathrm{a}$ \\
NGC 6826 & 0.10 & 4.8 & 0.58 & 3.95 & 1.5 & 2.6 & 1.4 & 8.5 & 8.0 & $\mathrm{~b}$ \\
IC 418 & $\geq 0.072$ & 6.2 & 0.95 & 3.5 & 0.88 & 4.4 & 1.8 & 12 & 8.8 & $\mathrm{c}$ \\
IC2448 & 0.094 & 2.7 & 0.55 & 2.5 & 0.64 & 2.0 & 1.2 & & 8.0 & $\mathrm{~d}$ \\
NGC 2392 & 0.080 & 3.3 & 1.85 & 2.9 & 0.85 & 5.0 & 2.2 & 13 & 8.4 & $\mathrm{e}$ \\
NGC 3242 & 0.092 & 1.95 & 1.0 & 3.8 & 0.90 & 2.8 & 1.7 & 7.0 & 8.1 & $\mathrm{f}$ \\
\hline Solar & $(0.085)$ & 2.7 & 0.675 & 4.9 & $(0.84)$ & 13. & $(2.5)$ & 31. & 8.0 & $\mathrm{~g}$ \\
\hline NGC 6302 & 0.17 & 0.6 & 2.9 & 2.3 & 2.2 & 7.8 & 6.0 & 34. & 6.4 & $\mathrm{~h}$ \\
NGC 6537 & 0.149 & 1.7 & 4.5 & 1.8 & 1.7 & 11. & 4.1 & 24. & 6.0 & $\mathrm{j}$ \\
\hline
\end{tabular}

References. a) Present paper; b) Surendiranath \& Pottasch (2008, A\&A, 483, 519); c) Pottasch et al. (2004, A\&A, 423, 593); d) Guiles, S., et al. (2007, ApJ 669, 1282); e) Pottasch et al. (2008, A\&A, 481, 393); f) Pottasch \& Bernard-Salas (2008, A\&A, 490, 715); g) Grevesse et al. (2010, Ap\&SS, 328, 179); h) Pottasch et al. (1999, A\&A, 347, 975); j) Pottasch et al. (2000, A\&A, 363, 767).

\subsubsection{Modeling and its implications}

Although we had tried a series of numerical model computations for this object by choosing various options in the parameter space, the primary road-block we faced was this: the observed fluxes of [O IV] $25.88 \mu \mathrm{m}$ and the He II $1640 \AA$ \&ere too high to reproduce while those of specie $\mathrm{O}_{\text {II }}, \mathrm{S}_{\text {II }}$ and $\mathrm{Cl}$ III were too low to be reproduced by the models. Many years ago, Aller (1987) modelled this object and he modified the incident energy spectrum to get a good fit. Later Adam \& Koeppen (1985, hereafter AK) had to introduce energy from stellar wind at a $T_{\mathrm{e}}$ of $3 \times 10^{5} \mathrm{~K}$ to adequately reproduce the observations. While Aller's adoptation is purely ad hoc, that of AK is not viable physics-wise. AK had predicted that such an extra source of energy should make the PN an X-ray emitter but this is not the case as shown by Guerrero et al. (2000). Secondly they claim that this additional energy source produces a good model matching observations. But looking at their final results, (see Table 13 of their paper) we find that they did not match the diagnostic lines of $\mathrm{O}$ II and $\mathrm{S}_{\text {II }}$ well. They did not include the optical lines of [ $\left.\mathrm{Cl}_{\mathrm{III}}\right]$, He II $1640 \AA$ and [O Iv] $25.88 \mu \mathrm{m}$ among others, whereas we used a comprehensive set of multi-wavelength spectral observations in this work. A more important point is that they claim that a proper nebular model with rigourous radiative transfer would match the observations very well. On the other hand, we have tried models having proper windy model atmospheres (for the CSPN) with Cloudy (wherein radiative transfer is handled by escape probability), and it did not work out well. We have even tried to infuse additional photons below $226 \mathrm{~A}$ in the input stellar radiation but this did not work out. It is clear that AK's suggestion of wind plasma as the extra energy source is ruled out.

As mentioned above $\mathrm{He}_{\mathrm{II}}$ and [O IV] lines are very strong and to produce them we needed a model atmosphere with a $T_{\text {eff }}$ of $120000 \mathrm{~K}$. But all other lines then do not match properly with such a model. We experimented with a whole range of values of the CSPN parameters but did not succeed. We note that from FUV observations of the continua of the CSPN by FUSE, Herald $\&$ Bianchi (2004) (HB hereafter) obtain a $T_{\text {eff }}$ of only $66000 \mathrm{~K}$. When we ran a model with the CSPN parmeters as given by HB, it did not reproduce the nebular spectra well. Diagnostic lines did not match; He II and [O IV] lines were weak. More importantly HB give a luminosity of around $4000 L_{\odot}$ which when used in our model, gave the transmitted flux as nearly $80 \%$ of the incident flux. We found that a luminosity of $550 L_{\odot}$ would be sufficient to produce the absolute $\mathrm{H}_{\beta}$ flux correctly. But only a high luminosity of $4000_{\odot}$ is compatible with the observed FUV continua of the CSPN. This creates another problem because this $\mathrm{PN}$ is quite complex as it shows hydrogen molecular absorption lines in its FUSE spectrum. These $\mathrm{H}_{2}$ molecules are attributed to be circum-nebular rather than interstellar by HB. It would be very difficult to imagine the presence of these molecules when the nebula leaks the incident stellar radiation enormously in the FUV and UV wavelengths! They would simply be destroyed by such a strong radiation. The dust content is not very high in this $\mathrm{PN}$ as shown by $\mathrm{HB}$ and so the idea of dust grains shielding $\mathrm{H}_{2}$ molecules from the strong radiation is also ruled out. In our modeling experiments we have even tried including a binary CSPN by way of introducing two sources of input radiation, a hot and a cooler star. We experimented with different combinations of temperature and luminosity but were not able to reproduce the observations.

In summary, the best we could intuitively guess was that since the complicated ionization strucuture as demanded by the observations of nebular emission lines was impossible to reproduce, it is probable that the wind streaming into the nebular material is directly injecting highly ionized elements, particularly $\mathrm{O}$ and $\mathrm{He}$. We are led to think of such a scenario under the given context. We feel that this PN is quite complicated as far as its physics and chemistry are concerned. It is important that this new idea of "wind streaming" is observationally explored to establish its possibility, especially as it may be applicable to other PNe which are difficult to model, such as NGC 2392. Shocks have been suggested as the source of energy needed to increse the ionization in NGC 2392 and NGC 1535 (e.g. Peimbert et al. 1995). But if the shocks result in a hot plasma as assumed by Adam \& Koeppen (1985) they are in disagreement with the observations as discussed above. Thus the suggestion of "ion streaming" is a good possibility for both NGC 2392 and NGC 1535. We conclude that the determination of abundances is possible, at present, only by the ICF method for this PN.

\section{Discussion}

In Table 17 the abundances for the four PNe discussed above are summarized in the first four lines, followed by the abundances for five other nebulae which have already been determined. As mentioned in the introduction all these PNe have been discussed by Mendez et al. $(1988,1992)$ and form a very homogeneous 
Table 18. Prediction of central star mass and distance from evolution theory.

\begin{tabular}{lcccccccccc}
\hline \hline PNe & $\begin{array}{c}T_{\text {eff }} \\
\mathrm{K}\end{array}$ & $m_{v}$ & $C$ & $\begin{array}{c}\text { Vel. } \\
\mathrm{km} \mathrm{s}^{-1}\end{array}$ & $\begin{array}{c}\text { Rad. } \\
\prime \prime\end{array}$ & $\begin{array}{c}t \\
10^{3} \mathrm{~s}\end{array}$ & $R_{\mathrm{s}} / R_{\odot}$ & $L_{\mathrm{s}} / L_{\odot}$ & $\begin{array}{c}\text { Mass } \\
M_{\odot}\end{array}$ & $\begin{array}{c}\text { Dist. } \\
\mathrm{kpc}\end{array}$ \\
\hline NGC1535 & 66000 & 12.11 & 0.08 & 20 & 10.5 & 5.3 & 0.543 & 5000 & 0.59 & 2.0 \\
Tc 1 & 32000 & 11.38 & 0.36 & 12.5 & 5.0 & 5,4 & 2.17 & 4500 & 0.57 & 2.64 \\
He2-108 & 32000 & 12.72 & 0.53 & 12 & 5.5 & 9.2 & 1.96 & 3550 & 0.56 & 3.7 \\
NGC 6629 & 46000 & 12.87 & 0.88 & 6.5 & 7.7 & 9.5 & 0.95 & 3800 & 0.57 & 1.8 \\
NGC 6826 & 48000 & 10.68 & 0.07 & 11 & 12.7 & 8.4 & 0.935 & 4080 & 0.57 & 1.42 \\
IC 418 & 36000 & 10.23 & 0.33 & 12 & 6 & 4.0 & 1.60 & 5000 & 0.60 & 1.25 \\
IC 2448 & 65000 & 14.26 & 0.27 & 13.5 & 5 & 6.5 & 0.54 & 4550 & 0.58 & 4.05 \\
NGC 2392 & 43000 & 10.63 & 0.22 & 53 & 22.4 & 1.7 & 1.55 & 7600 & 0.62 & 1.8 \\
NGC 3242 & 75000 & 12.32 & 0.12 & 27.5 & 19 & 4.7 & 0.43 & 5100 & 0.59 & 1.75 \\
\hline
\end{tabular}

group. Not only are these nebulae excited by bright, rather low temperature central stars which are rather far from the galactic plane, the central stars all have spectra indicating that they are hydrogen rich. Mendez (1991) classifies them all as either $\mathrm{O}(\mathrm{H})$ or $\mathrm{Of}(\mathrm{H})$.

As may be seen from the table, the abundances of these PNe are rather uniform. The oxygen abundance varies by a factor of 1.9 but some of this may be caused by the known abundance gradient with distance from the galactic plane. To better judge this effect, an approximate value of the distance of the PNe from the galactic center is given in Col. 10 of the table. The nitrogen abundance, or better still the N/O ratio also has the same low value for all the PNe, with the single exception of NGC 2392. This low value of N/O is the same as found in the solar atmosphere, which is also shown in Table 17. Also notice that the helium abundance of these PNe is very similar to the solar value. Combining these abundances with the theoretical determinations of Karakas (2003) we obtain the following picture. These PNe originate from stars of low enough mass so that the second dredge-up or hot-bottom burning have not taken place. These processes would have increased both the helium abundance and the N/O ratio to values higher than observed. For comparison the last two entries in Table 17 show the abundances of PNe whose central stars are of higher mass and have clearly undergone hotbottom burning.

The ratio of carbon to oxygen $(\mathrm{C} / \mathrm{O})$ clearly varies for the PNe shown in Table 17. The three lowest values are similar to the solar value, about 0.5 . The highest $\mathrm{C} / \mathrm{O}$ ratio, that of $\mathrm{IC} 418$ is almost four times as high. The $\mathrm{PNe}$ with the lowest $\mathrm{C} / \mathrm{O}$ ratio probably originate in stars of similar mass to the sun while the higher $\mathrm{C} / \mathrm{O}$ value originate in somewhat higher mass stars which have undergone the third dredge-up. Following Karakas (2003) the initial mass of the stars with the low carbon abundance is between 1 and $1.5 M_{\odot}$, while substantial carbon will be produced betweem 1.75 and $2.5 M_{\odot}$. According to Weidemann (2000) the first group corresponds to a final core mass of 0.55 to $0.57 M_{\odot}$, while the group with substantial carbon will have a final mass of between 0.59 and $0.63 M_{\odot}$.

The observed nebular abundances do not permit a more quantitative determination of the stellar masses than that given above. We can however, determine the stellar masses which are predicted by stellar evolution theory to see if they are consistent with the masses determined from the nebular abundances above. To do this we make use of the summary of stellar evolution calculations given by Blocker (1995) in the form of an HR diagram where the time of evolution from the AGB is marked on each evolutionary track (his Fig. 12). Lines of constant time (isochrones) are also shown in this figure. We have fixed the position of each of the central stars being discussed on this figure by determining the stellar temperature and age. The stellar temperature is taken from the spectra of the stars using the work of Mendez et al. (1988), Kudritzki et al. (2006) and Pauldrach et al. (2004), and is listed in Col. 2 of Table 18. In seven of the nine cases these temperatures are the same as are determined from the nebula (e.g. see Pottasch \& Bernard-Salas 2010). In two cases the spectroscopic temperature is lower: NGC 2392, where the difference is considerable, and NGC 1535, where the difference is much smaller. The reason for this difference is not yet understood, but is probably related to the heating of the nebula as described above.

The PN age is determined from the observed size and expansion velocity of the nebulae. These quantities have been taken from the values listed by Acker et al. (1992) and are given in Cols. 5 and 6 of the table. When two values of velocity are listed by Acker et al. (1992), the value for the [N II] is used since this line is formed farthest out in the nebula. The velocity is measured in the line-of-sight while the measured size is tangential but because these nebulae are nearly round it is expected that these values may be combined. Having determined the age of the nebula its position on the HR diagram is now fixed. The values of luminosity and core mass corresponding to this position are shown in Cols. 9 and 10 of Table 18. While uncertainties in the value of size and velocity may be considerable and the age determination only reliable to within $50 \%$, the values of luminosity and core mass found are much better determined because the isochrones are so closely spaced, i.e. the luminosity and core mass have a rather small dependance on the age in the low temperature range of the evolutionary tracks.

The core mass found in this way is the same as that deduced above from the nebular abundances. Even in the prediction that the $\mathrm{PNe}$ with the high $\mathrm{C} / \mathrm{O}$ ratios will have somewhat higher core masses appears to be fulfilled. We may say that the core masses predicted from the PNe abundances are consistent with those found from stellar evolution.

From the luminosity found (in Col. 9) and the assumption that the central star radiates as a blackbody with the temperature given in Col. 2 and the angular radius found from the stellar magnitude and extinction listed in Cols. 3 and 4, the distance can be computed. These distances are listed in the last column of Table 18. In 6 of the 9 cases this distance agrees to within $15 \%$ with the statistical distances given either by Cahn et al. (1992) or Stanghellini et al. (2008). In the remaining three cases (NGC 2392, NGC 3242 and IC 418) the listed distance is about $60 \%$ higher.

\section{Conclusions}

With the help of Spitzer infrared spectra the abundances in four $\mathrm{PNe}$ have been determined. These PNe are all excited by rather low temperature central stars and have similar morphological 
and kinematic properties: they are all nearly round and are rather far from the galactic plane. We are able to show that these nebulae have rather similar abundances of helium, oxygen, nitrogen, carbon and other elements. The resultant abundances are summarized in the first four lines of Table 17. We then show that five other PNe with low temperature central whose abundances have been determined using Spitzer infrared spectra and have the same or similar morphological and kinematic properties also have the same or similar abundances.

By comparing these abundances with those predicted by nucleosynthesis models by Karakas (2003) it is deduced that these PNe originate from stars of initial mass between $1 M_{\odot}$ and about $2.5 M_{\odot}$, which according to Weidemann (2000) correspond to core masses of between $0.56 M_{\odot}$ and $0.63 M_{\odot}$. These values of core masses are compared with those determined from stellar evolution theory using the observed temperature of the central star and the measured age of the nebula. The core masses thus found are consistent with each other. Two details reinforce this consistency. First, the higher core masses from the evolutionary theory are found in $\mathrm{PNe}$ which have high $\mathrm{C} / \mathrm{O}$ abundance ratios as the models of Karakas (2003) predict. Secondly the distances found from the stellar evolution are in general values expected from statistical distance scales.

There are a number of uncertainties which must still be considered. First, it is not understood why the central star temperature measured in NGC 2392 is so much lower than that found from the nebula. Second, distances found by some researchers are different than found here. For example, the distances given by Kudritzki et al. (2006) for 6 of the PNe listed are 50\% higher than we have found. This could be due to errors in their determination of the stellar gravity from uncertain line profiles. Furthermore the expansion distances found for two of the nebulae (NGC 3242 and IC 2448) are at least a factor of 2 lower than we have found here. This should be carefully considered in the future.

Acknowledgements. We duly acknowledge the use of SIMBAD and ADS in this research work. We have used the IUE spectra archive at the STSCI and we wish to thank the archive unit for the same. R.S. would like to acknowledge that a part of his research work was done when he was working at the Indian Inst. of Astrophysics, Bangalore. R.S. sincerely thanks his former colleagues J. S. Nathan and B. A. Varghese for help with S/W upgradation.

\section{References}

Acker, A., Marcout, J., Ochsenbein, F., et al. 1992, Strasbourg-ESO catalogue Adams, J., \& Koppen, J. 1985, A\&A, 142, 461

Aller, L. H. 1982, Ap\&SS, 83, 225

Aller, L. H., \& Czyzak, S. J. 1979, Ap\&SS, 62.397

Aller, L. H., \& Keyes, C. D. 1987, ApJS, 65, 405

Banerjee, D. P. K., \& Anandaro, B. G. 1991, A\&A, 250, 165

Barker, T. 1989, ApJ, 340, 421

Benjamin, R. A., Skillman, E. D., \& Smits, D. P. 1999, ApJ, 514, 307

Bernard Salas, J., Pottasch, S. R., Beintema, D. A., \& Wesselius, P. R. 2001, A\&A, 367, 949

Blocker, T. 1995, A\&A, 299, 755

Cahn, J. H., Kaler, J. B., \& Stanghellini, L. 1992, A\&AS, 94, 399

Cami, J., Bernard-Salas, J., Peeters, E., et al. 2010, Science 329, 1180

Ciardullo, R., Bond, H. E., \& Sipior, M. S. 1999, AJ, 118, 488
Corradi, R. L. M., Schonberner, D., Steffen, M., et al. 2003, MNRAS, 340, 417 Condon, J. J., \& Kaplan, D. L. 1998, ApJS, 117, 361

Davey, A. R., Storey, P. J., \& Kisielius, R. 2000, A\&AS, 142, 85

Feibelman, W. A. 1983, PASP, 95, 886

Fluks, M. A., Plez, B., de Winter, D., et al. 1994, A\&AS, 105, 311

de Freitas Pacheco, J. A., Maciel, W. J., \& Costa, R. D. D. 1992, A\&A, 261, 579

Gathier, R., \& Pottasch, S. R. 1988, A\&A, 197, 266

Gathier, R., Pottasch, S. R., \& Pel, J. W. 1986, A\&A, 157, 171

Grevesse, N., Asplund, M., Sauval, A. J., et al. 2010, Ap\&SS 328, 179

Griffith, M. R., wright, A. E., Burke, B. F., \& Ekers, R. D. 1994, ApJS, 90, 179

Gregory, P. C., Vavasour, J. D., Scott, W. K., et al. 1994, ApJS, 90, 173

Guerrero, M. A., Chu, Y.-H., \& Gruendl, R. A. 2000, ApJS, 129, 295

Guiles, S., Bernard-Salas, J., Pottasch, S. R., et al. 2007, ApJ, 660, 1282

Henry, R. B. C., Kwitter, K. B., \& Balick, B. 2005, AJ, 127, 2284

Herald, J. E., \& Bianchi, L. 2004, ApJ, 609, 378

Higdon, S. J. U., Devost, D., Higdon, J. L., et al. 2004, PASP, 116, 975

Houck, J. R., Appleton, P. N., Armus, L., et al. 2004, ApJS, 154, 18

Hummer, D. G., \& Storey, P. J. 1987, MNRAS, 224, 801

Karakas, A. I. 2003, Thesis, Monash Univ. Melbourne (see also Karakas, A., \& Lattanzio, J. C. 2007, PASA 24, 103)

Kerber, F., Mignani, R. P., Guglielmetti, F., et al. 2003, A\&A, 408, 1029

Kingsburgh, R. L., \& Barlow, M. J. 1994, MNRAS, 271, 257

Kingsburgh, R. L., \& English, J. 1992, MNRAS, 259, 635

Krabbe, A. C., \& Copetti, M. V. F. 2006, A\&A, 450, 159

Kudritzki, R. P., Mendez, R. H., Puls, J., et al. 1997, Proc. IAU Symp., 180, ed. Habing, \& Lamers, 64

Kudritzki, R.-P., Urbaneja, M. A., \& Puls, J. 2006, IAU Symp., 234, ed. Barlow, \& Mendez, 119

Lattanzio, J. 2003, IAU Symp. 209, ed. S. Kwok, M. Dopita, \& R. Sutherland, 73

McKenna, F. C., Keenan, F. P., Kaler, J. B., et al. 1996, PASP, 108, 610

Mendez, R. H. 1991, IAUS 145, 375

Mendez, R. H., Kudritzki, R. P., Herrero, A., et al. 1988, A\&A, 190, 113

Mendez, R. H., Kudritzki, R. P., \& Herrero, A. 1992, A\&A, 260, 329

Milingo, J. B., Kwitter, K. B., \& Henry, R. B. C., et al. 2002a, ApJS, 138, 279

Milingo, J. B., Henry, R. B. C., \& Kwitter, K. B. 2002b, ApJS, 138, 285

Milingo, J. B., Kwitter, K. B., \& Henry, R. B. C., et al. 2010, ApJ, 711, 619

Milne, D. K., \& Aller, L. H. 1975, A\&A, 38, 183

Milne, D. K., \& Aller, L. H. 1982, A\&AS, 50, 209

Napiwotzki, R. 2006, A\&A, 451, L27

Pauldrach, A. W. A., Hoffmann, T. L., \& Mendez, R. H. 2004, A\&A, 419, 111

Peimbert, M. 1978, IAU Symp., 76, 215

Peimbert, M., \& Torres-Peimbert, S. 1983, IAU Symp., 103, 233

Peimbert, M., Torres-Peimbert, S., \& Luridiana, V. 1995, Rev. Mex. AA, 31, 131

Phillips, J. P. 2003, MNRAS, 344, 501

Porter, R. L., Bauman, R. P., Ferland, G. J., et al. 2005, ApJ, 622, L73

Pottasch, S. R., \& Acker, A. 1989 A\&A, 221, 123

Pottasch, S. R., \& Beintema, D. A. 1999, A\&A, 347, 974

Pottasch, S. R., \& Bernard-Salas, J. 2010, A\&A, 517, 95

Pottasch, S. R., Wesselius, P. R., Wu, C. C., et al. 1977, A\&A, 54, 435

Pottasch, S. R., Dennefeld, M., \& Mo, J.-E. 1986a, A\&A, 155, 397

Pottasch, S. R., Preite-Martinez, A., Olnon, F. M., et al. 1986b, A\&A, 161, 363

Pottasch, S. R., Beintema, D. A., \& Feibelman, W. A. 2000, A\&A, 363, 767

Pottasch, S. R., Beintema, D. A., Bernard Salas, J., \& Feibelman, W. A. 2001, A\&A, 380, 684

Pottasch, S. R., Beintema, D. A., Bernard Salas, J., et al. 2002, A\&A, 393, 285

Preite-Martinez, A., \& Pottasch, S. R. 1983, A\&A, 126, 31

Rauch, T. 2003, A\&A, 403, 709

Schwarz, H. E., Corradi, R. L. M., \& Melnick, J. 1992, A\&AS, 96, 23

Stanghellini, L., Shaw, R. A., \& Villaver, E. 2008, ApJ, 689, 194

Stoy, R. H. 1933, MNRAS, 93, 588

Surendiranath, R., Pottasch, S. R., \& García-Lario, P. 2004, A\&A, 421, 1051

Tylenda, R., Siodmiak, N., Gorny, S. K., et al. 2003, A\&A, 405, 627

Torres-Peimbert, S., \& Peimbert. M.1977, RMxAA 2, 181

Weidemann, V. 2000, A\&A, 363, 647

Williams, R., Jenkins, E. B., Baldwin, J. A., et al. 2008, ApJ, 677, 1100

Wright, A. E., Griffith, M. R., Burke, B. F., \& Ekers, R. D. 1994, ApJS, 91, 111 